\title{
Imaging the dynamical atmosphere of the red supergiant Betelgeuse in the CO first overtone lines with VLTI/AMBER ${ }^{\star, \star \star}$
}

\author{
K. Ohnaka ${ }^{1}$, G. Weigelt ${ }^{1}$, F. Millour ${ }^{1,2}$, K.-H. Hofmann ${ }^{1}$, T. Driebe ${ }^{1,3}$, \\ D. Schertl ${ }^{1}$, A. Chelli ${ }^{4}$, F. Massi ${ }^{5}$, R. Petrov ${ }^{2}$, and Ph. Stee ${ }^{2}$ \\ ${ }^{1}$ Max-Planck-Institut für Radioastronomie, Auf dem Hügel 69, 53121 Bonn, Germany \\ e-mail: kohnaka@mpifr.de \\ 2 Observatoire de la Côte d'Azur, Departement FIZEAU, Boulevard de l'Observatoire, BP 4229, 06304 Nice Cedex 4, France \\ 3 Deutsches Zentrum für Luft- und Raumfahrt e.V., Königswinterer Str. 522-524, 53227 Bonn, Germany \\ ${ }^{4}$ Institut de Planétologie et d'Astrophysique de Grenoble, BP 53, 38041 Grenoble Cedex 9, France \\ 5 INAF-Osservatorio Astrofisico di Arcetri, Instituto Nazionale di Astrofisica, Largo E. Fermi 5, 50125 Firenze, Italy
}

Received 7 December 2010 / Accepted 12 March 2011

\begin{abstract}
Aims. We present one-dimensional aperture synthesis imaging of the red supergiant Betelgeuse ( $\alpha$ Ori) with VLTI/AMBER. We reconstructed for the first time one-dimensional images in the individual CO first overtone lines. Our aim is to probe the dynamics of the inhomogeneous atmosphere and its time variation.

Methods. Betelgeuse was observed between 2.28 and $2.31 \mu \mathrm{m}$ with VLTI/AMBER using the 16-32-48 m telescope configuration with a spectral resolution up to 12000 and an angular resolution of 9.8 mas. The good nearly one-dimensional $u v$ coverage allows us to reconstruct one-dimensional projection images (i.e., one-dimensional projections of the object's two-dimensional intensity distributions).

Results. The reconstructed one-dimensional projection images reveal that the star appears differently in the blue wing, line center, and red wing of the individual CO lines. The one-dimensional projection images in the blue wing and line center show a pronounced, asymmetrically extended component up to $\sim 1.3 R_{\star}$, while those in the red wing do not show such a component. The observed onedimensional projection images in the lines can be reasonably explained by a model in which the $\mathrm{CO}$ gas within a region more than half as large as the stellar size is moving slightly outward with $0-5 \mathrm{~km} \mathrm{~s}^{-1}$, while the gas in the remaining region is infalling fast with 20-30 $\mathrm{km} \mathrm{s}^{-1}$. A comparison between the CO line AMBER data taken in 2008 and 2009 shows a significant time variation in the dynamics of the CO line-forming region in the photosphere and the outer atmosphere. In contrast to the line data, the reconstructed one-dimensional projection images in the continuum show only a slight deviation from a uniform disk or limb-darkened disk. We derive a uniform-disk diameter of $42.05 \pm 0.05$ mas and a power-law-type limb-darkened disk diameter of $42.49 \pm 0.06$ mas and a limb-darkening parameter of $(9.7 \pm 0.5) \times 10^{-2}$. This latter angular diameter leads to an effective temperature of $3690 \pm 54 \mathrm{~K}$ for the continuum-forming layer. These diameters confirm that the near-IR size of Betelgeuse was nearly constant over the last 18 years, in marked contrast to the recently reported noticeable decrease in the mid-IR size. The continuum data taken in 2008 and 2009 reveal no or only marginal time variations, much smaller than the maximum variation predicted by the current three-dimensional convection simulations.

Conclusions. Our two-epoch AMBER observations show that the outer atmosphere extending to $\sim 1.3-1.4 R_{\star}$ is asymmetric and its dynamics is dominated by vigorous, inhomogeneous large-scale motions, whose overall nature changes drastically within one year. This is likely linked to the wind-driving mechanism in red supergiants.
\end{abstract}

Key words. infrared: stars - techniques: interferometric - supergiants - stars: late-type - stars: atmospheres stars: individual: Betelgeuse

\section{Introduction}

Red supergiants (RSGs) experience slow, intensive mass loss up to $10^{-4} M_{\odot} \mathrm{yr}^{-1}$, which is very important for understanding the final fate of massive stars. For example, our poor understanding of the RSG mass loss makes it difficult to estimate the mainsequence mass range of the progenitors of the Type IIP supernovae, which are the most common type of core-collapse supernovae. The mass loss also plays a significant role in the chemical

* Based on AMBER observations made with the Very Large Telescope Interferometer of the European Southern Observatory. Program ID: 082.D-0280 (AMBER Guaranteed Time Observation).

$\star \star$ Appendices are available in electronic form at

http://www . aanda.org enrichment of galaxies. Despite this importance, there are no satisfactory theories for the RSG mass loss at the moment, as stressed by Harper (2010).

Studies of the outer atmosphere, where the winds are accelerated, are a key to tackling this problem. The outer atmosphere of RSGs has complicated structures. The UV observations of the well-studied RSG Betelgeuse ( $\alpha$ Ori, M1-2Ia-Ibe) with the Hubble Space Telescope reveal that the hot ( 6000-8000 K) chromospheric plasma is more than twice as extended as the photosphere (Gilliland \& Dupree 1996). However, radio continuum observations with the Very Large Array show that much cooler ( 1000-3000 K) gas extends to several stellar radii (Lim et al. 1998), suggesting that the hot chromospheric plasma and cooler gas coexist. IR spectroscopic and interferometric studies 
of a few bright RSGs also show the presence of dense $\mathrm{H}_{2} \mathrm{O}$ gas in the outer atmosphere, the so-called "MOLsphere", extending to $\sim 1.3-2.0 R_{\star}$ with column densities on the order of $10^{20} \mathrm{~cm}^{-2}$ and temperatures of 1500-2000 K (e.g., Tsuji 2000a,b, 2006; Ohnaka 2004; Perrin et al. 2004, 2007). The nature of the cool gas in the outer atmosphere of Betelgeuse has also recently been probed with mid-IR [Fe II] emission lines (Harper et al. 2009a). Possibly the chromospheric plasma with a small filling factor is embedded in more abundant, cooler gas. This inhomogeneous, multi-component nature of the outer atmosphere is considered to play a crucial role in driving mass outflows in RSGs. There is observational evidence for the asymmetric, inhomogeneous nature of the circumstellar material. For Betelgeuse, Kervella et al. (2009) found a very faint plume extending to $\sim 6 R_{\star}$ in the nearIR, while a millimeter CO map shows a blob at $\sim 5^{\prime \prime}\left(\sim 235 R_{\star}\right)$ away from the star (Harper et al. 2009b). The emission of the $\mathrm{CO}$ fundamental lines near $4.6 \mu \mathrm{m}$ shows that the circumstellar envelope is approximately spherical within $3^{\prime \prime}\left(140 R_{\star}\right)$ but with signatures of mildly clumpy structures (Smith et al. 2009).

High-spectral and high-spatial resolution observations of strong IR molecular lines are ideal for probing the physical properties of the outer atmosphere. The near-IR interferometric instrument AMBER (Astronomical Multi-BEam combineR) at the Very Large Telescope Interferometer (VLTI) is well suited for this goal with its high spectral resolution up to 12000 and high spatial resolution down to 1-2 mas with the current maximum baseline of $130 \mathrm{~m}$. In 2008, we observed Betelgeuse in the CO first overtone lines near $2.3 \mu \mathrm{m}$ with AMBER (Ohnaka et al. 2009, hereafter Paper I). The high spectral resolution of AMBER allowed us to detect salient signatures of inhomogeneities in the individual CO lines and to spatially resolve the gas motions in a stellar photosphere (and also MOLsphere) for the first time other than the Sun.

However, in 2008, we obtained data only at six $u v$ points, which are insufficient for image reconstruction. In order to obtain a more complete picture of the dynamics of the inhomogeneous outer atmosphere, we carried out new AMBER observations of Betelgeuse with a better $u v$ coverage in 2009. In this second paper, we report on the first one-dimensional aperture synthesis imaging of Betelgeuse in the CO first overtone lines, as well as on time variation in the dynamics of the stellar atmosphere in an interval of one year. The paper is structured as follows. The AMBER observations, data reduction, and image reconstruction are outlined in Sect. 2. We describe the results about the time variations as well as the one-dimensional image reconstruction in Sect. 3. The modeling of the velocity field presented in Sect. 4 is followed by the discussion on the dynamics in the extended outer atmosphere (Sect. 5). Conclusions are presented in Sect. 6.

\section{Observations}

\subsection{AMBER observations and data reduction}

AMBER (Petrov et al. 2007) is the near-IR (1.3-2.4 $\mu \mathrm{m}$ ) spectro-interferometric instrument at VLTI, which combines three $8.2 \mathrm{~m}$ Unit Telescopes (UTs) or $1.8 \mathrm{~m}$ Auxiliary Telescopes (ATs). AMBER measures the amplitude of the Fourier transform - the so-called visibility or visibility amplitude and two observables that contain information about the phase of the object's Fourier transform: differential phase (DP) and closure phase $(\mathrm{CP})$. The DP roughly represents how the object's phase in a spectral feature deviates from that in the continuum. Nonzero DP represents information about the photocenter shift in a

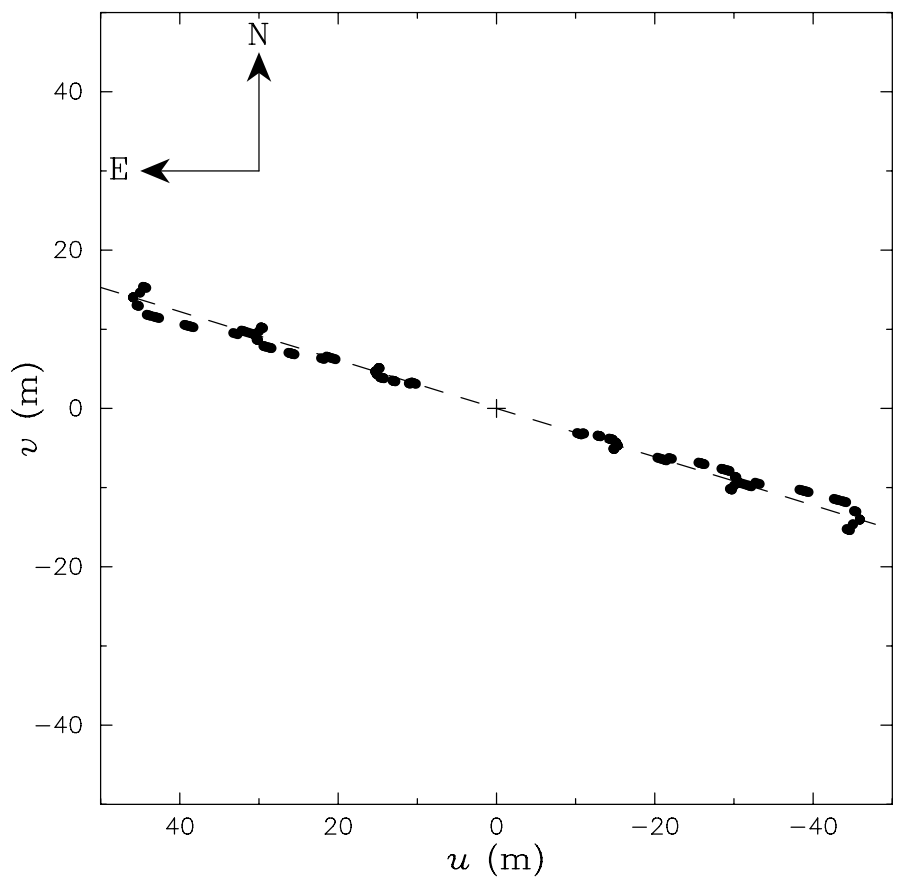

Fig. 1. $u v$ coverage of our AMBER observations of Betelgeuse. The dashed line represents the average position angle of $73^{\circ}$.

spectral feature with respect to the continuum. The CP is the sum of the measured Fourier phases around a closed triangle of baselines (i.e., $\varphi_{12}+\varphi_{23}+\varphi_{31}$ ), not affected by the atmospheric turbulence. The CP is always zero or $\pi$ for point-symmetric objects, and non-zero and non- $\pi$ CPs indicate an asymmetry in the object. Moreover the $\mathrm{CP}$ is important for aperture synthesis imaging in optical/IR interferometry.

Betelgeuse was observed on 2009 January 5 and 6 with AMBER using three ATs in the E0-G0-H0 linear array configuration with 16-32-48 m baselines (AMBER Guaranteed Time Observation, Program ID: 082.D-0280, P.I.: K. Ohnaka). As in Paper I, we used the $K$-band high-resolution mode (HR_K) with a spectral resolution of 12000 covering wavelengths from 2.28 to $2.31 \mu \mathrm{m}$ to observe the strong CO first overtone lines near the $(2,0)$ band head. Fringes could be detected on all three baselines without the VLTI fringe tracker FINITO. We obtained a total of 54 data sets on two half nights. The data sets taken more than $\sim 2$ min apart were treated as separate data sets, because Betelgeuse is strongly resolved and the visibility varies noticeably even for a slight change in the baseline length (see Fig. 2). As shown in Fig. 1, the observed $u v$ points align approximately linearly at position angles of $73 \pm 2^{\circ}$. This linear $u v$ coverage allows us to sample the visibility function densely at this particular position angle and to reconstruct one-dimensional projection images as described in Sect. 2.2. Each data set consists of 500 or 1000 frames (NDIT) with each frame taken with a detector integration time (DIT) of $120 \mathrm{~ms}$. Sirius ( $\alpha \mathrm{CMa}, \mathrm{A} 1 \mathrm{~V}$, $K=-1.4$ ) was observed for the calibration of the interferometric data of Betelgeuse. We adopted the same angular diameter of $5.6 \pm 0.15$ mas from Richichi \& Percheron (2005) as adopted in Paper I. We only used the calibrator data sets obtained just before and after each data set on Betelgeuse. A summary of the observations is given in Table A.1. 
K. Ohnaka et al.: Imaging the dynamical outer atmosphere of Betelgeuse in the CO first overtone lines

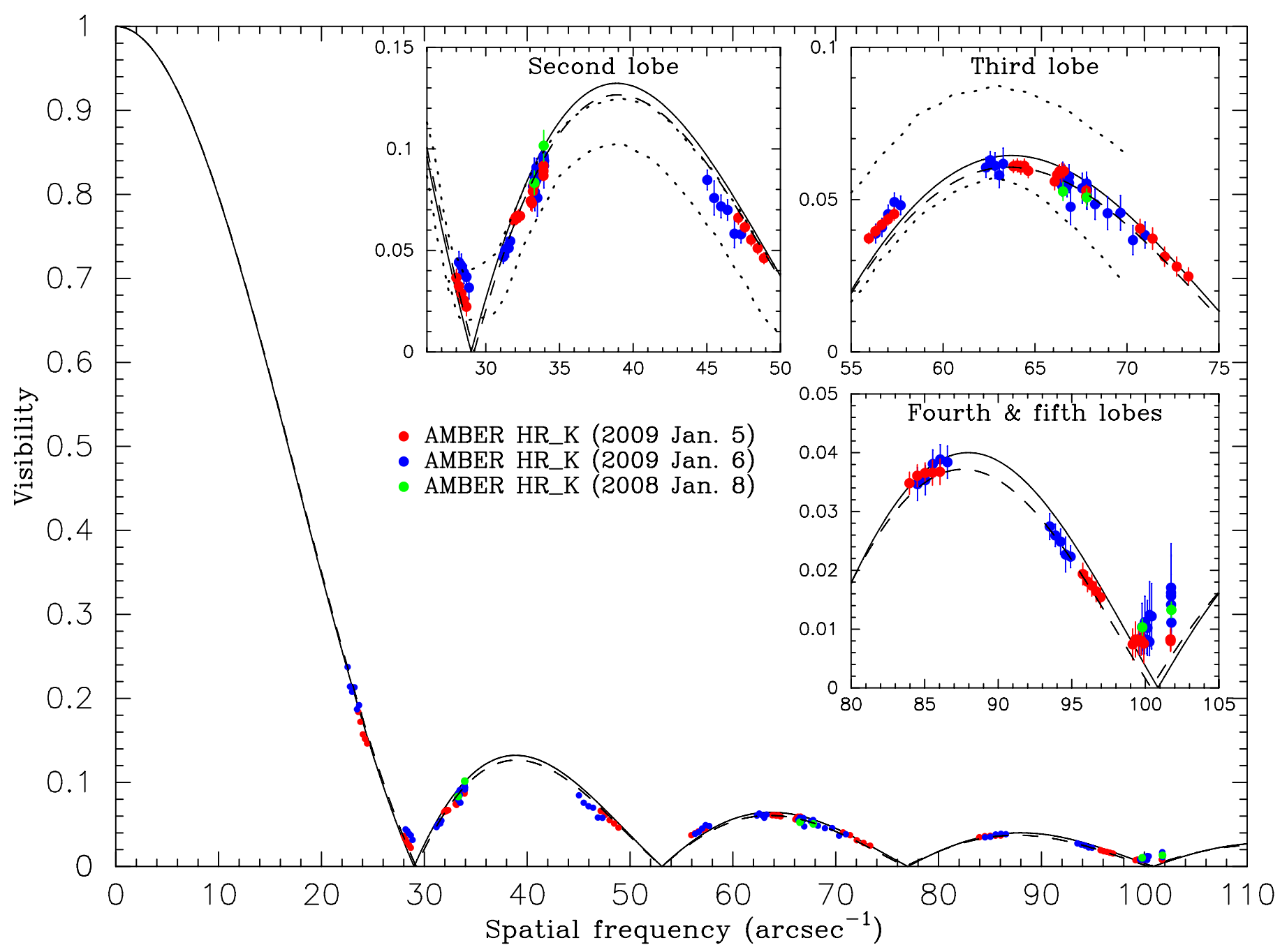

Fig. 2. Continuum visibilities of Betelgeuse averaged over the continuum spectral channels between 2.28 and $2.293 \mu \mathrm{m}$. The insets show enlarged views of the second, third, and fourth/fifth lobes. The solid and dashed lines represent the visibilities for a uniform disk with a diameter of 42.05 mas and for a limb-darkened disk with a diameter of 42.49 mas and a limb-darkening parameter of 0.097 (power-law-type limb-darkened disk of Hestroffer 1997), respectively. The dotted lines represent the maximum range of the variations in the $2.22 \mu \mathrm{m}$ visibility due to timedependent inhomogeneous surface structures predicted by the three-dimensional convection simulation of Chiavassa et al. (2009), who presents the model prediction up to $70 \operatorname{arcsec}^{-1}$.

We reduced our AMBER data with amdlib ver.2.21, which is based on the P2VM algorithm (Tatulli et al. 2007). Some data sets, particularly those measured on the longest baseline and/or near the $\mathrm{CO}$ band head, are too noisy for the analysis. Therefore, we improved the SNR by binning the entire raw data (object, dark, sky, and P2VM calibration data) in the spectral direction with a running box car function as described in Paper I. We used different binnings with these spectral resolutions:

1. spectral resolution $=12000$ (no binning), 8000, and 4800 for the data sets \#16, \#17, \#18, and \#45-\#49 for comparison with the 2008 data (Sects. 3.1 and 3.3);

2. spectral resolution $=6000$ for all data sets for the image reconstruction in the continuum and in the individual, isolated CO lines (Sects. 3.4 and 3.5, Fig. 5);

\footnotetext{
1 Available at http://www.jmmc.fr/data-processing_amber. htm
}

3. spectral resolution $=1600$ for all data sets for the image reconstruction near the $C O$ band head at $2.294 \mu \mathrm{m}$ (Sect. 3.6, Fig. 6).

We checked for a systematic difference in the calibrated visibilities and differential/closure phases by taking the best $20 \%, 50 \%$, and $80 \%$ of all frames in terms of the fringe SNR (Tatulli et al. 2007). The difference between the results obtained with the best $20 \%$ and $80 \%$ frames is typically $\sim 10 \%$. We took the best $20 \%$ of all frames for our final visibilities to avoid the systematic bias due to the rapid atmosphere and to keep decent SNRs in the final results. On the other hand, the calibrated differential/closure phases do not show this systematic dependence on the frame selection criterion. Therefore, we included the best $80 \%$ of all frames for the final DPs and CPs. The errors of the resulting visibilities, DPs, and CPs were estimated in the same manner as in Paper I.

We removed telluric lines from the observed spectra of Betelgeuse as best as possible by using Sirius as a spectroscopic standard star. The telluric lines identified in the spectrum of 
Sirius were also used for wavelength calibration. As a template of the telluric lines, we convolved the atmospheric transmission spectra measured at the Kitt Peak National Observatory ${ }^{2}$ to match the spectral resolutions of the data. The uncertainty in wavelength calibration is $2.0 \times 10^{-5} \mu \mathrm{m}\left(2.6 \mathrm{~km} \mathrm{~s}^{-1}\right)$. We note that the uncertainties in wavelength calibration for the 2008 data and 2006 data in Paper I were mistakenly overestimated. The correct uncertainties in the 2008 data and 2006 data are $2.3 \times 10^{-5} \mu \mathrm{m}\left(3.0 \mathrm{~km} \mathrm{~s}^{-1}\right)$ and $1.8 \times 10^{-4} \mu \mathrm{m}\left(25.1 \mathrm{~km} \mathrm{~s}^{-1}\right)$, respectively.

\subsection{One-dimensional image reconstruction}

The good linear $u v$ coverage along the position angle of $73^{\circ}$ shown in Fig. 1 provides an opportunity to reconstruct the socalled one-dimensional projection image, which is obtained by integrating the object's two-dimensional intensity distribution along the direction perpendicular to the linear $u v$ coverage on the sky (central slice theorem or Fourier slice theorem). In other words, this one-dimensional projection image represents the two-dimensional intensity distribution compressed or squashed onto the linear $u v$ coverage on the sky. For example, the onedimensional projection image of a uniform disk is a semi-circle (see also the two-dimensional image of a limb-darkened disk and its one-dimensional projection image shown in Figs. B.1a and c). The reconstruction of one-dimensional projection images was first proposed for radio interferometry by Bracewell (1956). Whereas the information in the direction perpendicular to the baseline vector is lost in one-dimensional projection images, they still provide model-independent information about the geometrical extent and asymmetry of the object. The reconstruction of one-dimensional projection images from IR interferometric data or lunar occultation data has been carried out (e.g., Navarro et al. 1990; Leinert et al. 1991; Tatebe et al. 2006; Chandler et al. 2007).

We used the MiRA package ver. $0.9 .9^{3}$ (Thiébaut et al. 2008) to reconstruct one-dimensional projection images at each spectral channel (details of our image reconstruction procedure are described in Appendix B). We first carried out the image reconstruction using computer-simulated data to examine effects of the $u v$ coverage and reconstruction parameters such as the initial model, prior, and regularization scheme on the reconstructed images. These tests with simulated data are crucial for examining the credibility of aperture synthesis imaging particularly for objects with complex structures.

With appropriate reconstruction parameters determined from these tests, we attempted to reconstruct one-dimensional projection images from the observed 162 visibility amplitudes and 54 CPs. While this worked well for the continuum, the reconstruction in the $\mathrm{CO}$ lines turned out to be very sensitive to the reconstruction parameters. For example, depending on the size of the uniform disk used as the initial model, the reconstructed one-dimensional projection image in the $\mathrm{CO}$ lines shows a faint region on the eastern or western side. Therefore, we used the self-calibration technique, which has recently been successfully applied to AMBER data for the first time by Millour et al. (2011). We added modifications to their technique to deal with some issue specific to our data of Betelgeuse as described in Appendix C. This technique allows us to restore the phase of the

\footnotetext{
2 http://www.eso.org/sci/facilities/paranal/ instruments/isaac/tools/spectra/atmos_S_K.fits

3 http://www-obs.univ-lyon 1 . fr/labo/perso/eric. thiebaut/mira.html
}

complex Fourier transform of the object's intensity distribution from the DP measurements. Image reconstruction with the complex visibility (i.e., visibility amplitude and phase) removes the ambiguity of the solution derived with the visibility amplitude and $\mathrm{CP}$ alone.

\section{Results}

\subsection{Continuum data}

To compare with the $K$-band continuum visibilities from the 2008 data that were derived from the binned data with a spectral resolution 4800, we also derived the visibilities from the 2009 data binned with the same spectral resolution. As in Paper I, we selected continuum points shortward of the CO band head at $2.294 \mu \mathrm{m}$. For each data set, we averaged the visibilities over the selected continuum points. We took the simple mean of the errors as the errors in the average continuum visibilities without reducing by $\sqrt{N_{\text {cont }}}$, where $N_{\text {cont }}$ is the number of the selected continuum points. The reason is that the measurement errors are dominated by the systematic error in the absolute visibility calibration and do not become smaller by the averaging. We applied this averaging to the 2008 data as well. Since the different continuum spectral channels correspond to slightly different spatial frequencies, we also averaged the spatial frequencies from the selected continuum points.

Figure 2 shows the $K$-band continuum visibilities measured in 2008 and 2009 as a function of spatial frequency. The figure reveals that the nearly linear $u v$ coverage shown in Fig. 1 enabled us to sample the visibility function quite densely from the first to the fifth visibility lobe. Uniform-disk fitting to the 2009 data results in a diameter of $42.05 \pm 0.05$ mas with a reduced $\chi^{2}$ of 3.8. Fitting with a power-law-type limb-darkend disk (Hestroffer et al. 1997) results in a limb-darkened disk diameter of $42.49 \pm$ 0.06 mas and a limb-darkening parameter of $(9.7 \pm 0.5) \times 10^{-2}$ with a better reduced $\chi^{2}$ of 2.5 . While the reduced $\chi^{2}$ value is still higher than 1, Fig. 2 shows that the deviation from the limbdarkened disk is not strong, as found for the 2008 data. Only at the highest spatial frequency (i.e., the smallest spatial scale) is the deviation noticeable, but the errors are also large there.

The limb-darkened disk diameter derived from the $K$-band continuum data and a bolometric flux of $(111.67 \pm 6.49) \times$ $10^{-13} \mathrm{~W} \mathrm{~cm}^{-2}$ (Perrin et al. 2004) lead to an effective temperature of $3690 \pm 54 \mathrm{~K}$. We propose this value as an effective temperature of the continuum-forming layer, approximately free from the effects of molecular lines. Perrin et al. (2004) modeled $K$-broadband interferometric measurements of Betelgeuse with a continuum-forming blackbody sphere and an extended molecular shell. They derived $3690 \pm 50 \mathrm{~K}$ for the continuum-forming sphere. This value excellently agrees with our effective temperature of the continuum-forming layer. Our effective temperature is slightly higher than the $3600 \pm 66 \mathrm{~K}$ recently derived by Haubois et al. (2009) from the the $H$-band observations with the Infrared Optical Telescope Array (IOTA), but both agree within the uncertainties.

Figure 2 reveals that the continuum visibilities show no or only marginal time variations between 2008 (green dots) and 2009 (red and blue dots) within the measurement errors. We compare this observational result with the current threedimensional convection simulation for RSGs by Chiavassa et al. (2009). The visibility predicted for $2.2 \mu \mathrm{m}$ (Fig. 18 of Chiavassa et al. 2009), which approximately samples the continuum, shows maximum time variations of, for example, $\pm 40 \%$ in the third lobe. However, the visibilities observed in the third lobe (at 
spatial frequencies of $66-68 \operatorname{arcsec}^{-1}$ ) show no time variation within the error bars (3-5\%), and these error bars are 8-13 times smaller than the predicted maximum variation. While it is not very likely that we observed Betelgeuse at two epochs when it accidentally showed the same, weak deviations from the limb-darkened disk, this possibility cannot be entirely excluded. However, it is also possible that the current three-dimensional convection simulation predicts too pronounced surface structures and time variations owing to the gray approximation adopted for the radiative transfer, as Chiavassa et al. (2009) mention. The most direct test for three-dimensional convection simulations is to measure the amplitude of the temporal fluctuations in the visibility and closure phase as well as the time scale of fluctuations by long-term monitoring observations and compare these with the model predictions. AMBER observations at more epochs would be necessary to draw a definitive conclusion about whether or not Betelgeuse seen in the continuum indeed shows much weaker inhomogeneities and much smaller time variations than predicted by the current three-dimensional convection simulation.

The deviation from the limb-darkened disk in the $K$-band continuum visibilities is lower than that observed in the $H$ broadband by Haubois et al. (2009). Their measurements show deviations of the visibilities from the limb-darkened disk as high as $80-120 \%$ already in the fourth lobe (converted from the squared visibilities plotted in their Fig. 4), where our $K$-band continuum data still follow the limb-darkened disk within the measurement errors of 5-10\% except for the data points near the visibility null at $\sim 100 \mathrm{arcsec}^{-1}$. The cause of this difference is not yet clear, because of a number of differences between their observations and ours (e.g., differences in the observed wavelengths, spectral resolution, position angle coverage). $H$-band observations with higher spectral resolution and/or $K$-band observations with a wider position angle coverage are necessary to clarify this issue.

\subsection{Long-term behavior of the near-IR and mid-IR angular diameters}

Figure 3 shows the $K$-band uniform-disk diameters of Betelgeuse from the literature and the archival data summerized in Paper I, together with the $11 \mu \mathrm{m}$ uniform-disk diameter presented in Townes et al. (2009), who found a noticeable decrease in the $11 \mu \mathrm{m}$ size in the last 15 years, and the one-epoch measurement by Perrin et al. (2007). In marked contrast to the noticeable decrease in the $11 \mu \mathrm{m}$ size, the $K$-band diameter has been quite stable for the last 18 years with only a possible, slight long-term decrease. These results can be qualitatively explained as follows. While the size of the star itself has been stable over the last 18 years, the temperature and densities and/or shape of the outer atmosphere have changed significantly (e.g., decrease in temperature and/or density). Because the mid-infrared apparent size is largely affected by the MOLsphere and by dust (Ohnaka 2004; Verhoelst et al. 2006; Perrin et al. 2007), the changes in the outer atmosphere lead to a noticeable change in the $11 \mu \mathrm{m}$ size. This interpretation has also been recently reached by Ravi et al. (2010) based on the estimation of the surface temperature seen at $11 \mu \mathrm{m}$. On the other hand, the angular size measured with the $K$-broadband filter is only slightly affected, because the strong molecular bands of $\mathrm{CO}$ and $\mathrm{H}_{2} \mathrm{O}$ are present only at the short and long wavelength edge of the $K$ band. Detailed modeling of the mid-IR interferometric and spectroscopic data, which is necessary to quantitatively derive the change in the physical properties of the MOLsphere and dust,

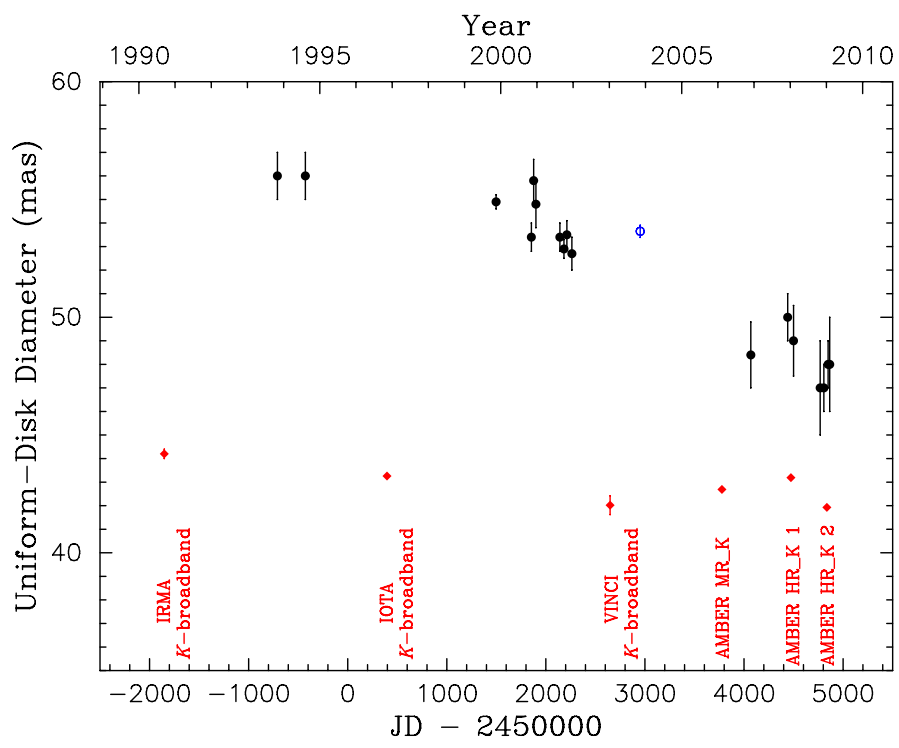

Fig. 3. Uniform-disk diameters of Betelgeuse measured in the $K$ band and at $11 \mu \mathrm{m}$ as a function of time. The black filled circles and the blue open circle represent the $11 \mu \mathrm{m}$ diameters measured by Townes et al. (2009) and Perrin et al. (2007), respectively. The diamonds represent the $K$-band diameters from the following references. IRMA: Dyck et al. (1992). IOTA: Perrin et al. (2004). VINCI: Paper I. AMBER MR_K: AMBER medium-resolution $(\lambda / \Delta \lambda=1500)$ data from Paper I. AMBER HR_K 1: AMBER high-resolution data from Paper I. AMBER HR_K 2: present paper. The errors in the IOTA and AMBER measurements are smaller than the symbols.

is beyond the scope of this paper and will be pursued in a forthcoming paper.

\subsection{Significant time variation in the CO first overtone lines}

Figure 4 shows a comparison between the data taken in 2008 and 2009 for four representative CO lines. The results for the 2009 data were obtained from the merged data of the data sets $\# 16$, \#17, and \#18, which were taken at uv points very close to one of the data sets obtained in 2008 (data set \#1 in Paper I). The data sets \#45-\#49 were also taken at $u v$ points very close to the same 2008 data set, and the results from these data sets agree well with those shown in Fig. 4. We used the binning with the same spectral resolution as applied to the data from 2008: spectral resolution of 12000 (no binning) for the $16 \mathrm{~m}$ baseline data, 8000 for the $32 \mathrm{~m}$ baseline data, and 4800 for the $48 \mathrm{~m}$ baseline data and $\mathrm{CP}$, respectively.

In marked contrast to the continuum data, Fig. 4 reveals significant time variations in the $\mathrm{CO}$ line visibilities. The visibility within each CO line obtained on the $16 \mathrm{~m}$ baseline in 2008 was characterized by the maxima in the blue wing and minima in the red wing (black line in Fig. 4b). In the 2009 data, the visibility on the $16 \mathrm{~m}$ baseline does not show the maxima in the blue wing anymore (red line in Fig. 4b) and is characterized only by the minima in the red wing. The visibilities on the $32 \mathrm{~m}$ and $48 \mathrm{~m}$ baselines also show time variations, although the data on the $48 \mathrm{~m}$ baseline are noisy.

Time variations are even clearer in the DPs and CPs. Nonzero DPs were not detected in the CO lines on the $16 \mathrm{~m}$ baseline in 2008, but the 2009 data show clear non-zero DPs in the CO lines. On the other hand, the non-zero DPs on the $32 \mathrm{~m}$ baseline obtained in 2009 are much weaker than those in the 2008 data. The DPs on the $48 \mathrm{~m}$ baseline as well as the CPs measured in 2009 also show significant time variations. The non-zero DPs 

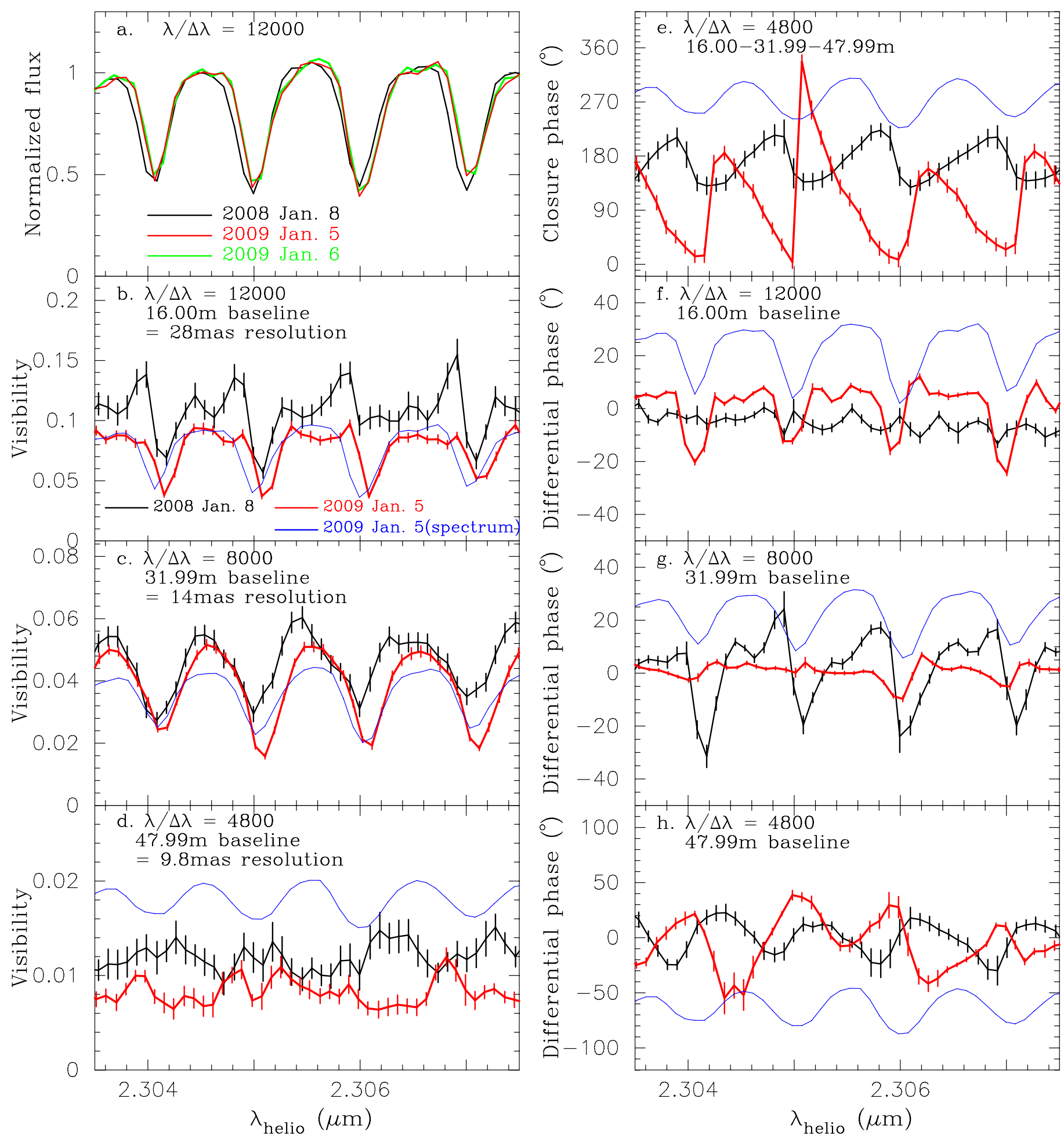

Fig. 4. AMBER data in the CO first overtone lines obtained in 2008 and 2009. The black and red solid lines represent the data taken in 2008 and 2009 , respectively. In the panels b)-h), the scaled spectrum observed on 2009 Jan. 5 is overplotted by the blue solid line to show the asymmetry of the visibilities and differential/closure phases within each line profile. The spectral resolutions given in the panels are the values of the binned data as described in Sect. 2.1. a) Normalized spectra. Two spectra derived from the data taken on 2009 Jan. 5 and 6 are plotted by the red and green solid lines, respectively, while the 2008 spectrum is plotted by the black solid line. b)-d) Visibilities observed on the E0-G0-16 m, G0-H0-32 m, and E0-H0-48 m baselines. e) Closure phase. f)-h) Differential phases observed on the E0-G0-16 m, G0-H0-32 m, and E0-H0-48 m baselines.

and non-zero/non- $\pi$ CPs indicate the asymmetry of the CO-lineforming region in 2009, as found in 2008.

The observed spectra also reveal changes in the line profiles. The lines observed in 2009 are redshifted by $\sim 6 \mathrm{~km} \mathrm{~s}^{-1}$ compared to those observed in 2008, as shown in Fig. 4a. The spectra taken on 2009 Jan. 5 and 6 agree very well, although they were calibrated independently. This confirms that the redshift of the CO lines in the 2009 data is real. All these results suggest that the dynamics in the atmosphere of Betelgeuse has changed in an interval of one year. 


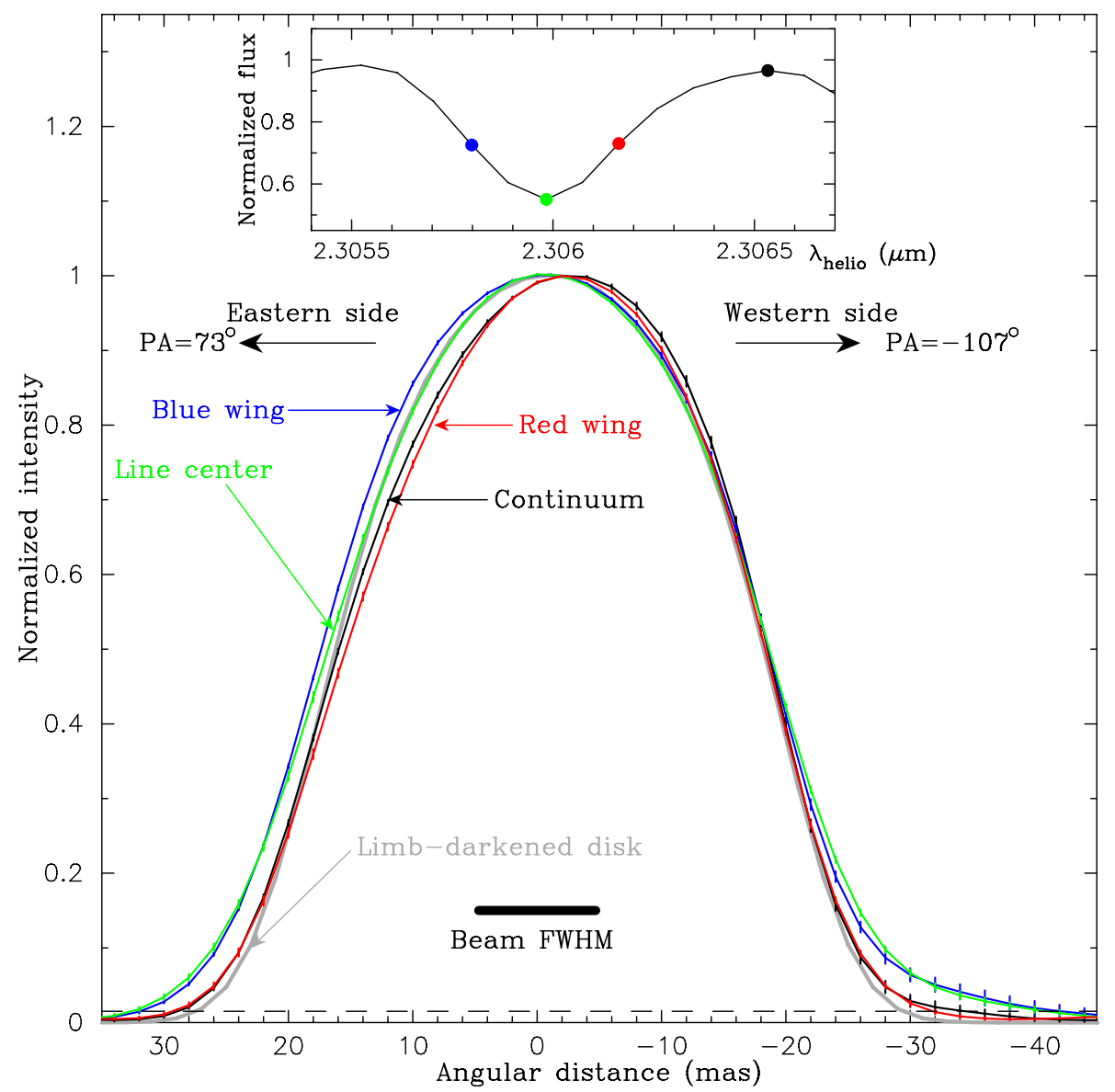

Fig. 5. One-dimensional projection images reconstructed at four different wavelengths (blue wing, line center, red wing, and continuum) within the $\mathrm{CO}$ line due to the two transitions $(2,0) R(26)$ and $R(75)$ (spectral resolution of 6000) are shown by the blue, green, red, and black solid lines, respectively. The one-dimensional projection image of the limbdarkened disk with the parameters derived in Sect. 3.1 is also shown by the gray solid line as a reference. The inset shows the observed line profile with the wavelengths of the images marked by the filled circles with the corresponding colors. The one-dimensional projection images are convolved with the Gaussian beam whose $F W H M$ ( 9.8 mas) is shown by the thick solid line, and their absolute scale is normalized to the peak intensities. The orientation of the one-dimensional projection images is also shown. The dashed line denotes the noise level of the image reconstruction.

\subsection{One-dimensional projection images in the continuum}

The reconstructed one-dimensional projection image in the continuum at $2.3065 \mu \mathrm{m}$ is shown by the black line in Fig. 5 (comparison between the observed interferometric data and those from the reconstructed image is shown in Fig. D.1). Also plotted is the one-dimensional projection image of the limb-darkened disk with the angular diameter of 42.49 mas and the limbdarkening parameter of 0.097 (gray line, overlapping with the green line at angular distances between +15 and -15 mas). These one-dimensional projection images are already convolved with the Gaussian beam with a $F W H M$ of 9.8 mas as described in Appendix B. Figure 5 shows that the stellar surface is well resolved with the beam size of $1 / 4$ of the diameter of the stellar disk. The one-dimensional projection images obtained at all continuum spectral channels agree well within the uncertainty of the reconstruction $(\sim 1 \%)$.

The overall deviation from the limb-darkened disk is small, $\sim 5 \%$ on the eastern side (position angle $=73^{\circ}$ ). Because inhomogeneities in the direction perpendicular to the baseline vector on the sky are smeared out in the one-dimensional projection image, we estimated the upper limit on the strength of inhomogeneities using a uniform disk with one Gaussian-shaped dark spot. The $H$-band image of Betelgeuse shows spots with FWHMs up to 10-11 mas (Haubois et al. 2009), which we adopt for our model. For a given amplitude of the spot, we generated 10000 models with random positions of the spot and counted the number of models whose one-dimensional projection image shows deviation from the uniform disk smaller than $5 \%$. For spot amplitudes lower than $20 \%$ of the stellar disk intensity, more than $70 \%$ of the models show deviations compatible with the observations. However, the fraction of these models is $13 \%$,
$3 \%$, and $<0.01 \%$ for spot amplitudes of $30 \%, 40 \%$, and $50 \%$ of the stellar disk intensity, respectively. Therefore, we estimate the amplitude of the spot to be smaller than $20-30 \%$ of the stellar disk intensity with flux contributions of $1.6-2.4 \%$.

\subsection{One-dimensional projection images in the individual CO lines}

Figure 5 shows the one-dimensional projection images reconstructed in the blue wing, line center, and red wing within the CO line centered at $2.306 \mu \mathrm{m}$ because of the two transitions $(2,0) R(26)$ and $R(75)$ with a spectral resolution of 6000 (comparison between the observed interferometric quantities and those from the reconstructed images is shown in Fig. D.1). The one-dimensional projection images are normalized with the peak intensities but are not artificially registered with one another, because the relative astrometry is preserved thanks to the restored visibility phase. The round or blunt shape of the images primarily results from the projection of the two-dimensional images onto the baseline vector on the sky. The level of the image reconstruction noise is estimated to be $\sim 1.5 \%$ from the strengths of the artifacts in the entire field of view used for the reconstruction (256 mas). The one-dimensional projection images in other, isolated CO lines above $\sim 2.3 \mu \mathrm{m}$ agree well with those shown in Fig. 5, which adds fidelity to the image reconstruction. These one-dimensional projection images represent the imaging of the photosphere and MOLsphere of an RSG, for the first time, in the individual CO first overtone lines.

Clearly, the one-dimensional projection images in the blue wing and line center are more extended than those in the continuum, with the extension of $15 \%$ and $30 \%$ on the eastern and 


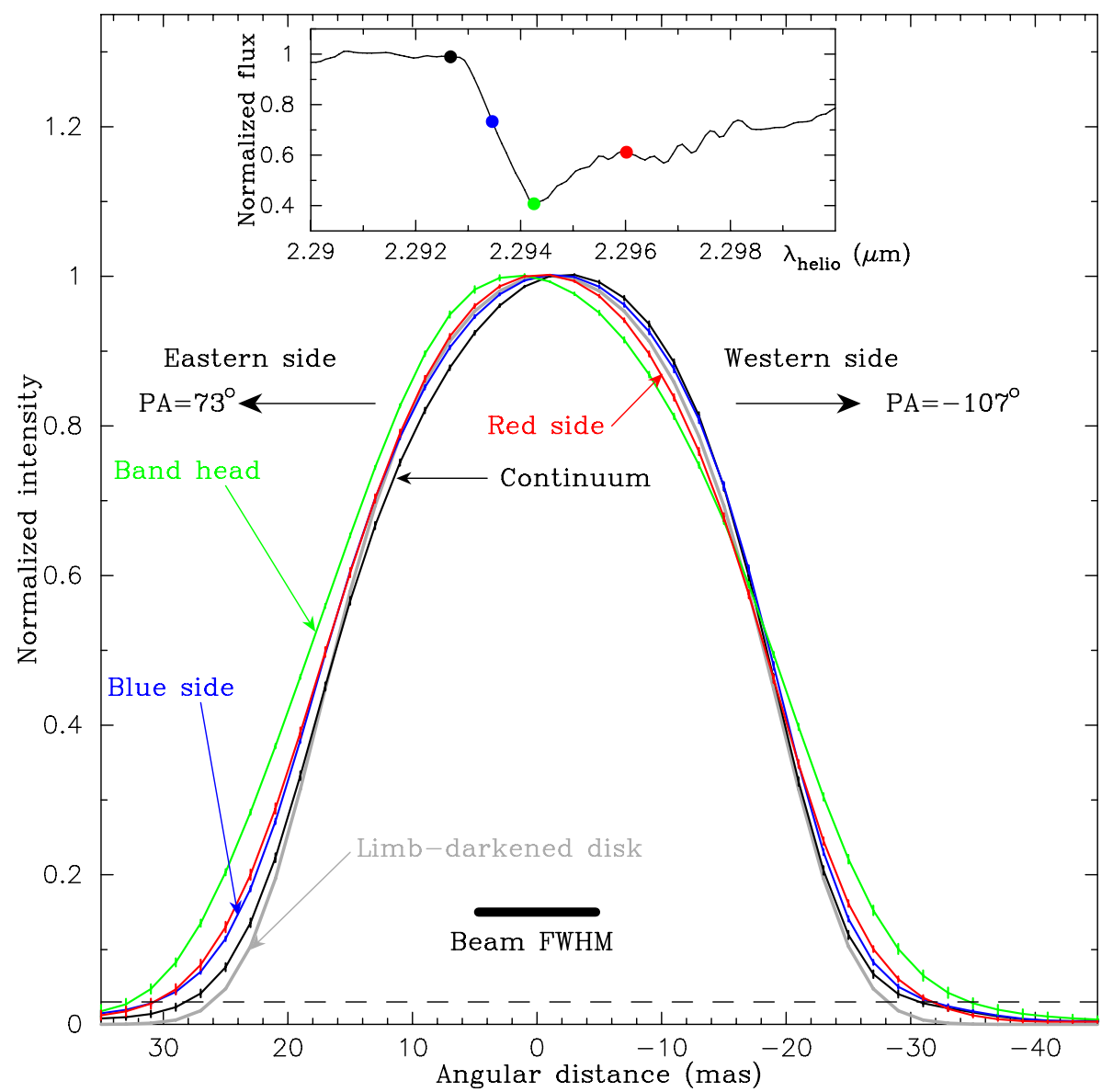

Fig. 6. One-dimensional projection images reconstructed at four different wavelengths in the $\mathrm{CO}(2,0)$ band head (spectral resolution of 1600) shown in the same manner as Fig. 5. western side, respectively, when measured at the noise level of the image reconstruction $(\sim 1.5 \%$ of the peak intensities). The actual geometrical extension with intensities lower than the reconstruction noise level could be even larger. These values are roughly consistent with the size of the MOLsphere probed with the $\mathrm{H}_{2} \mathrm{O}$ bands (e.g., Tsuji 2000a, 2006; Ohnaka 2004; Perrin et al. 2004, 2007). On the other hand, the red wing one-dimensional projection image shows only a slight deviation from the continuum without a trace of the extended component. Furthermore, the different appearance of the extended component in the blue and red wing suggests that the vigorous, inhomogeneous gas motions are present not only in the photosphere extending to $\sim 1.1 R_{\star}$ (see Sect. 4 in Paper I) but also in the layers extending to $\sim 1.3 R_{\star}$. This is because if the inhomogeneous gas motions were present only in the photosphere, the extended component would appear the same in the blue and red wing.

\subsection{One-dimensional projection images in the CO band head}

Figure 6 shows the one-dimensional projection images reconstructed at four different wavelengths in the $\mathrm{CO}(2,0)$ band head with a spectral resolution of 1600 (see Fig. D.2 for a comparison between the observed interferometric quantities and those from the reconstructed images). The peak of the one-dimensional projection image at the band head is shifted to the east with respect to that in the continuum and has geometrical extensions of $18 \%$ and $13 \%$ on the eastern and western side, respectively, when measured at the noise level of the image reconstruction.

In none of the reconstructed images in the continuum, $\mathrm{CO}$ lines, and $\mathrm{CO}$ band head did we detect a feature corresponding to the faint plume reported by Kervella et al. (2009), although their (single-dish) observations were carried out coincidentally almost simultaneously with our AMBER measurements. This is presumably because of the very faint nature of the plume. Its intensity is below $1 \%$ of the center of the stellar disk even at $\$ 1.3 \mu \mathrm{m}$, where it appears the most prominent, and the plume is even less pronounced at 2.12 and $2.17 \mu \mathrm{m}$. Such a faint structure is below the noise of the image reconstruction from the present data $(1.5 \%$ and $3 \%$ in Figs. 5 and 6, respectively). However, future observations with better accuracies could reveal the presence of the plume in the CO lines, which would be useful for understanding the nature of the plume. Furthermore, the reconstructed images in the $\mathrm{CO}$ band head with a spectral resolution 1600 can be compared with future medium-spectral resolution AMBER observations.

\section{Modeling of the velocity field}

We used our stellar patch model presented in Paper I to characterize the velocity field in the photosphere and MOLsphere. This model consists of two CO layers that represent the photosphere and MOLsphere. An inhomogeneous velocity field is represented by a patch (or clump) of $\mathrm{CO}$ gas moving at some velocity different from the $\mathrm{CO}$ gas in the remaining region. Because the reconstructed one-dimensional projection images do not allow us to know the actual number and shape of the patches, we assumed only one patch in our modeling to keep the number of free parameters as small as possible. Furthermore, for the temperature, $\mathrm{CO}$ column density, and the radius of the two layers, we used the same parameters as derived in Paper I for the following reason. As discussed in Sect. 3, the CO line profiles observed 

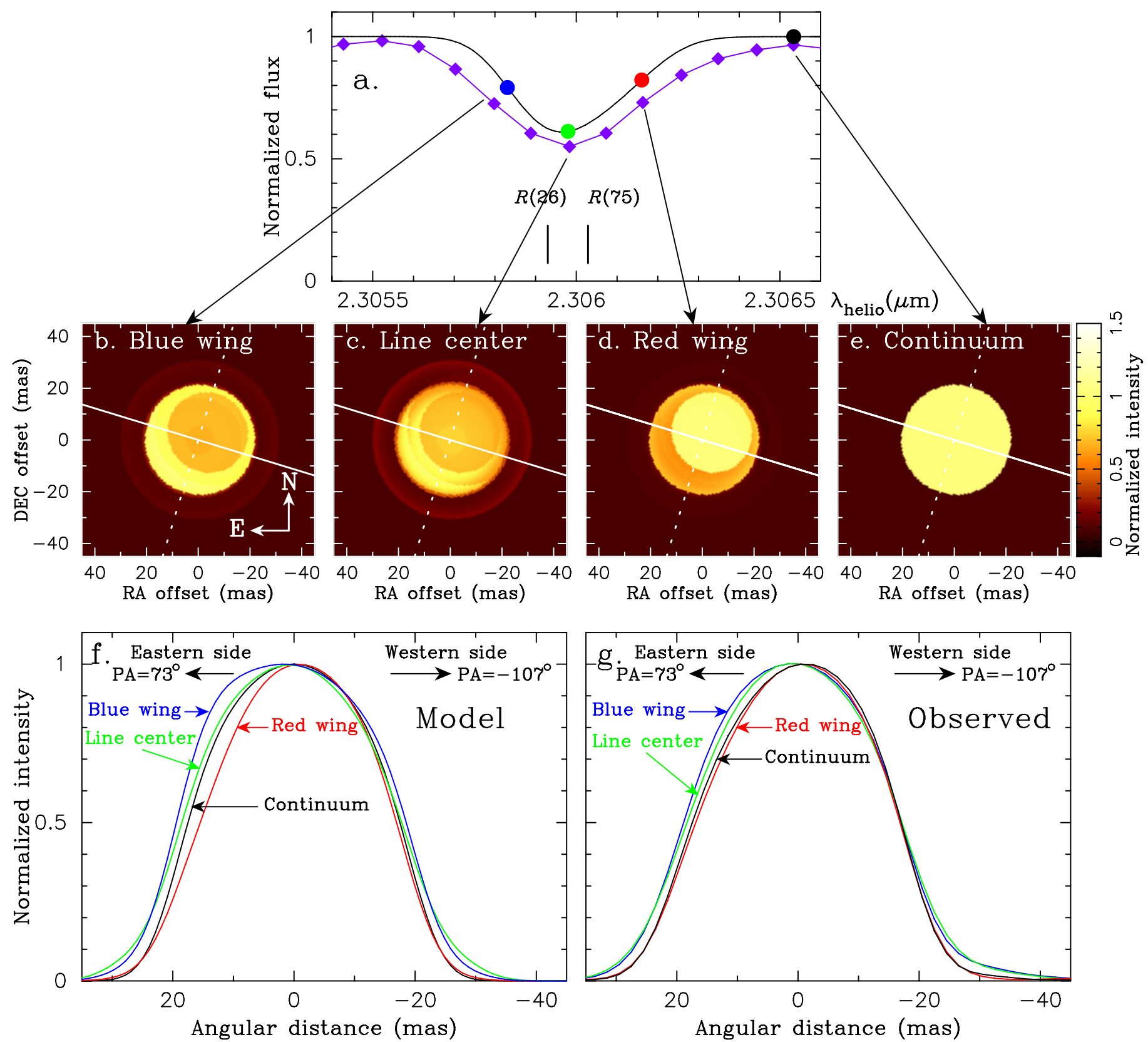

Fig. 7. Best-fit stellar patch model with an inhomogeneous velocity field. a) The filled diamonds represent the observed spectrum, while the black solid line represents the model spectrum. The filled circles mark the wavelengths of the two-dimensional images and one-dimensional projection images shown in the panels b)-g). b)-e) Two-dimensional model images. The orientation of the linear $u v$ coverage is shown by the solid lines. The one-dimensional projection images are obtained by integrating the two-dimensional images in the direction shown by the dotted lines, which is perpendicular to the orientation of the linear $u v$ coverage. f) The one-dimensional projection images predicted by the model at four wavelengths within the CO line. g) The observed one-dimensional projection images for the same CO line.

in 2008 and 2009 show little time variation in the line depth and width except for the redshift. This implies that the physical properties of the photosphere and MOLsphere, such as the density and temperature, may not have changed significantly, although there must have been temporal and spatial fluctuations. The parameters adopted from Paper I are as follows: the inner CO layer is assumed to be located at $1.05 R_{\star}$ with a temperature of $2250 \mathrm{~K}$ and a CO column density of $5 \times 10^{22} \mathrm{~cm}^{-2}$, while the outer CO layer is assumed to be located at $1.45 R_{\star}$ with a temperature of $1800 \mathrm{~K}$ and a CO column density of $1 \times 10^{20} \mathrm{~cm}^{-2}$. We also adopted a microturbulent velocity of $5 \mathrm{~km} \mathrm{~s}^{-1}$ for both layers as in Paper I. This means that we attempt to explain the onedimensional projection images observed in 2009 by changes in the velocity field, as well as in the position and size of the patch. The wavelength scale of the model spectra was converted to the heliocentric frame assuming a heliocentric velocity of $20 \mathrm{~km} \mathrm{~s}^{-1}$ (Huggins 1987; Huggins et al. 1994).

Figure 7 shows the two-dimensional images, onedimensional projection images, and the spectrum predicted by the best-fit model for the same $\mathrm{CO}$ line as shown in Fig. 5. The model is characterized by a large, off-centered, circular patch of CO gas, which dominates the upper half of the stellar disk (Figs. $7 \mathrm{~b}$ and $\mathrm{d}$ ). The $\mathrm{CO}$ gas within this patch is moving outward with a velocity of $5 \mathrm{~km} \mathrm{~s}^{-1}$, while the gas in the remaining region is moving inward faster with $25 \mathrm{~km} \mathrm{~s}^{-1}$. Figures $7 \mathrm{a}$ and $\mathrm{f}$ show that the line profile and the wavelength 
dependence of the observed one-dimensional projection images within the $\mathrm{CO}$ line are reasonably reproduced, although the difference between the images in the blue wing and red wing is somewhat too pronounced, and the line profile is weaker than the observed data. We found out that the models with a patch of $\mathrm{CO}$ gas moving slowly outward at $0-5 \mathrm{~km} \mathrm{~s}^{-1}$ with the gas in the remaining region downdrafting much faster at $20-30 \mathrm{~km} \mathrm{~s}^{-1}$ can reproduce the observed one-dimensional projection images reasonably.

The above model can also explain why the MOLsphere in the blue wing is much more pronounced than in the red wing. Firstly, the velocity field with the weak upwelling and strong downdrafting components causes the line center to be redshifted with respect to the stellar rest frame. This can be seen in Fig. 7a, where the positions of the two transitions responsible for the observed line profile, $(2,0) R(26)$ and $R(75)$, are marked in the stellar rest frame. Since the contribution of the $R(26)$ transition to the line profile is much larger than that of $R(75)$ because of the much lower excitation potential of the former transition, the line profile is redshifted with respect to the position of $R(26)$ in the stellar rest frame. In other words, the wavelength of the stellar rest frame is located in the blue wing of the line. Secondly, the strong extended $\mathrm{CO}$ emission is seen in the line of sight tangential to the outer $\mathrm{CO}$ layer, because the column density along this line of sight is the largest. The radial velocity of the CO layer along such a line of sight is nearly zero, which means that the strong extended $\mathrm{CO}$ emission appears at the stellar rest frame. Because the stellar rest frame is located in the blue wing as explained above, the extended $\mathrm{CO}$ emission is strong in the blue wing. The emission becomes nearly absent in the red wing, because the velocity difference between the blue and red wing is much greater than the line width.

The velocity field in 2009 is in contrast with that in 2008, which was characterized by the gas moving both outward and inward with velocities of $10-15 \mathrm{~km} \mathrm{~s}^{-1}$. Therefore, our AMBER observations at two epochs reveal a drastic change in the velocity field in the photosphere and MOLsphere within one year.

\section{Discussion}

The drastic change in the velocity field between 2008 and 2009 sets an upper limit of one year on the time scale of the change of the MOLsphere. This allows us to estimate the upper limit of the radial spatial scale where the inhomogeneous gas motions are present. We assume that the upwelling patch (or clump) with $10-15 \mathrm{~km} \mathrm{~s}^{-1}$ detected at $1.45 R_{\star}$ (radius of the MOLsphere) in 2008 decelerated linearly with time over one year and corresponds to the patch slowly moving outward with $0-5 \mathrm{~km} \mathrm{~s}^{-1}$ in 2009. Then the maximum radial distance reached by the gas patch is $0.24-0.47 R_{\star}$. This means that the upwelling gas patch at $1.45 R_{\star}$ in 2008 can reach $1.7-1.9 R_{\star}$ in 2009. Likewise, if we assume that the fast downdrafting patch with $20-30 \mathrm{~km} \mathrm{~s}^{-1}$ detected in 2009 accelerated inward linearly with time starting from $0 \mathrm{~km} \mathrm{~s}^{-1}$, it must have traveled $0.71-1.07 R_{\star}$ in 1 year. This suggests that the fast downdrafting gas patch could have been located as far as at 2.2-2.5 $R_{\star}$ in 2008 and could have fallen to $1.45 R_{\star}$ in one year. Therefore, the vigorous gas motions can be present up to $\sim 2 R_{\star}$.

These inhomogeneous gas motions in the extended atmosphere of Betelgeuse have also been detected by other observations. Recently Harper et al. (2009a) have studied the dynamics of the cool extended outer atmosphere of Betelgeuse based on high-spectral resolution mid-IR observations of the [Fe II] lines at 17.94 and $24.53 \mu \mathrm{m}$. These [Fe II] lines form at $\sim 1.6 R_{\star}$ (converted with the angular diameter of Betelgeuse derived here) in the cool extended outer atmosphere (see Fig. 8 of Harper et al. 2009 a) with estimated excitation temperatures of $1520-1950 \mathrm{~K}$. Therefore, the [Fe II] lines originate in the region similar to the MOLsphere where the CO first overtone lines form. The profiles of the [Fe II] lines indicate turbulent gas motions without signatures of significant outflows of $\gtrsim 10 \mathrm{~km} \mathrm{~s}^{-1}$. This is consistent with the velocity fields derived from our two-epoch AMBER observations. Harper et al. (2009a) detected no significant changes in the [Fe II] line profiles at three epochs over 14 months. However, this may be because the changes in the velocity field are smeared out in their spatially unresolved observations. As can be seen in Fig. 4a, the CO line profiles observed with AMBER only show a low redshift, despite the remarkable change in the velocity field.

Complex gas motions have been detected in the extended chromosphere of Betelgeuse as well. Lobel \& Dupree (2001) present the modeling of the chromospheric velocity field up to $\sim 3 R_{\star}$. Moreover, the velocity field changed from overall inward motions to outward motions within $0.5-1$ year. Therefore, both the cool and hot components in the extended outer atmosphere are characterized by strongly temporally variable inhomogeneous gas motions.

The physical mechanism responsible for these vigorous motions and their drastic change within one year is not yet clear, although it is likely related to the unknown wind-driving mechanism. The convective energy flux is expected to be low in the MOLsphere, which extends to $\sim 1.3-1.4 R_{\star}$. This poses a problem for the interpretation of the detected gas motions in terms of convection. Other possible mechanisms include Alfvén waves and pulsation. The recent detection of magnetic fields in Betelgeuse, albeit weak $(\sim 1 \mathrm{G})$, indicates that the prerequisite for Alfvén-driven-winds is available (Aurière et al. 2010). Airapetian et al. (2000) show that Alfvén waves can drive mass outflows from the chromosphere with the velocity and mass-loss rate in agreement with those observed for Betelgeuse. However, the effects of the Alfvén-waves on the more dominant, cool outer atmosphere including the CO MOLsphere are not addressed. The MHD simulations of Suzuki (2007) for red giants show that the stellar winds are highly temporally variable and "structured", in which hot $\left(\gtrsim 10^{4} \mathrm{~K}\right)$ gas bubbles are embedded in cool ( $\sim 2000 \mathrm{~K})$ gas (see, however, Airapetian et al. 2010, for critical discussion). The radial velocity within $\sim 10 R_{\star}$ also shows significant time variations from $\sim+40 \mathrm{~km} \mathrm{~s}^{-1}$ (outward motions) to $\sim-40 \mathrm{~km} \mathrm{~s}^{-1}$ (inward motions). This is compatible to the change in the velocity field detected by our AMBER observations. However, the simulations of Suzuki (2007) were carried out for red giant stars, which are much less luminous $\left(\$ 10^{3} L_{\odot}\right)$ compared to Betelgeuse $\left(1.3 \times 10^{5} L_{\odot}\right.$, Harper et al. 2008). Extending the MHD simulations of Suzuki (2007) for more luminous stars, as well as the inclusion of the cool molecular component in the work of Airapetian et al. (2000), would be valuable for a comparison with the present and future AMBER observations.

Lobel (2010) infers that strong shock waves generated by convection in the photosphere that are propagating outward may carry the energy and momentum to accelerate the wind and heat the chromosphere. The qualitative similarity between the inhomogeneous velocity field in the chromosphere and in the photosphere/MOLsphere may point toward this scenario. However, obviously, it is indispensable to map the dynamical structure of the cool outer atmosphere at various radii to clarify the driving mechanism of mass outflows in RSGs. 


\section{Concluding remarks}

We have succeeded, for the first time, in one-dimensional aperture synthesis imaging of Betelgeuse in the individual $\mathrm{CO}$ first overtone lines, as well as in the continuum approximately free from molecular/atomic lines, with a spatial resolution of 9.8 mas and a spectral resolution of 6000 using VLTI/AMBER.

The one-dimensional projection images in the $\mathrm{CO}$ lines reconstructed with the self-calibration technique, which restores the complex visibility using differential phase measurements, reveal that the star appears different within the individual CO lines. The one-dimensional projection images in the blue wing and line center show a pronounced extended component up to $1.3 R_{\star}$, while the images in the red wing follow that in the continuum without an extended component. Our image reconstruction represents the first study to image the so-called MOLsphere of an RSG in the individual CO first overtone lines. Our modeling suggests that the dynamics in the photosphere and MOLsphere in 2009 is characterized by strong downdrafts with $20-30 \mathrm{~km} \mathrm{~s}^{-1}$ and slight outward motions with $0-5 \mathrm{~km} \mathrm{~s}^{-1}$. This indicates a drastic change in the velocity field within one year from 2008, when the dynamics was characterized by both upwelling and downdrafting components with $10-15 \mathrm{~km} \mathrm{~s}^{-1}$.

On the other hand, the reconstructed one-dimensional projection images in the $K$-band continuum show only a small deviation of 5\% from the limb-darkened disk with an angular diameter of $42.49 \pm 0.06$ mas with a power-law-type limb-darkening parameter of $(9.7 \pm 0.5) \times 10^{-2}$. This limb-darkened disk diameter results in an effective temperature of $3690 \pm 54 \mathrm{~K}$ for the continuum-forming layer. The deviation from the limb-darkened disk in the one-dimensional projection images suggests that the amplitude of stellar spots is likely smaller than 20-30\% of the intensity of the stellar disk. Furthermore, we detected no or only marginal time variation in the continuum visibility data within the measurement errors, much smaller than the maximum variation predicted by the current three-dimensional convection simulations. It cannot be entirely excluded that Betelgeuse showed unusually weak surface structures at the times of our AMBER observations just by chance. However, it is also possible that the current three-dimensional convection model for RSGs predicts too strong surface structures in the continuum. A long-term monitoring to measure the amplitude of the time variations in the visibility and closure phase is indispensable for a definitive, statistical test of three-dimensional convection simulations.

The self-calibration imaging using differential phase has turned out to be very effective and necessary, despite the good linear $u v$ coverage from the first to fifth visibility lobe. This suggests that the self-calibration technique may be even more necessary for two-dimensional imaging, where it is difficult to obtain a $u v$ coverage as densely sampled as in our one-dimensional case. While the imaging of stellar surfaces is still challenging (e.g., Creech-Eakman et al. 2010), our self-calibration onedimensional imaging demonstrates a promising way to achieve that goal.

Acknowledgements. We thank the ESO VLTI team for supporting our AMBER observations. We are also grateful to Eric Thiébaut, who kindly makes his image reconstruction software MiRA publicly available. NSO/Kitt Peak FTS data on the Earth's telluric features were produced by NSF/NOAO.

\section{References}

Airapetian, V. S., Ofman, L., Robinson, R. D., Carpenter, K., \& Davila, J. 2000, ApJ, 528, 965

Airapetian, V. S., Carpenter, K. G., \& Ofman, L. 2010, ApJ, 723, 1210

Aurière, M., Donati, J.-F., Konstantinova-Antova, R., et al. 2010, A\&A, 516, L2

Bracewell, R. N. 1956, Aust. J. Phys., 9, 198

Chandler, A. A., Tatebe K., Hale, D. D. S., \& Townes, C. H. 2007, ApJ, 657, 1042

Chiavassa, A., Plez, B., Josselin, E., \& Freytag, B. 2009, A\&A, 506, 1351

Creech-Eakman, M., Young, J., Haniff, C., et al. 2010, SPIE, 7734E, 106

Dyck, H. M., Benson, J. A., Ridgway, S. T., \& Dixon, D. J. 1992, AJ, 104, 1982

Gilliland, R. L., \& Dupree, A. K. 1996, ApJ, 463, L29

Goorvitch, D. 1994, ApJS, 95, 535

Harper, G. M. 2010, Hot and Cool: Bridging Gaps in Massive Star Evolution, ASP Conf. Ser., 425, 152

Harper, G. M., Brown, A., \& Guinan, E. 2008, AJ, 135, 1430

Harper, G. M., Richter, M. J., Ryde, N., et al. 2009a, ApJ, 701, 1464

Harper, G. M., Carpenter, K. G., Ryde, N., et al. 2009b, AIP Conf. Ser., 1094, 868

Haubois, X., Perrin, G., Lacour, S., et al. 2009, A\&A, 508, 923

Hestroffer, D. 1997, A\&A, 327, 199

Huggins, P. J. 1987, ApJ, 313, 400

Huggins, P. J., Bachiller, R., Cox, P., \& Forveille, T. 1994, ApJ, 424, L127

Kervella, P., Verhoelst, T., Ridgway, S. T., et al. 2009, A\&A, 504, 115

Leinert, Ch., Haas, M., Richichi A., Zinnecker, H., \& Mundt, R. 1991, A\&A, 250,407

Lim, J., Carilli, C., White, S. M., Beasley, A. J., \& Marson, R. G. 1998, Nature, 392,575

Lobel, A. 2010, ASP Conf. Ser., 425, 162

Lobel, A., \& Dupree, A. K. 2001, ApJ, 558, 815

Millour, F., Meilland, A., Chesneau, O., et al. 2011, A\&A, 526, A107

Navarro, R., Benítez, D., \& Javier Fuentes, F. 1990, J. Opt. Soc. Am. A, 7, 459

Ohnaka, K. 2004, A\&A, 421, 1149

Ohnaka, K., Hofmann, K.-H., Benisty, M., et al. 2009, A\&A, 503, 183

Perrin, G., Ridgway, S. T., Coudé du Foresto, V., et al. 2004, A\&A, 418, 675

Perrin, G., Verhoelst, T., Ridgway, S. T., et al. 2007, A\&A, 474, 599

Petrov, R. G., Malbet, F., Weigelt, G., et al. 2007, A\&A, 464, 1

Ravi, V., Wishnow, E., Lockwood, S., \& Townes, C. 2010, Proc. 16th Cambridge Workshop on Cool Stars, Stellar Systems, and the Sun, ASP. Conf. Ser., in press [arXiv: 1012.0377]

Richichi, A., \& Percheron, I. 2005, A\&A, 434, 1201

Smith, N., Hinkle, K. H., \& Ryde, N. 2009, AJ, 137, 3558

Suzuki, T. K. 2007, ApJ, 659, 1592

Tatebe, K., Chandler, A. A., Hale, D. D. S., \& Townes, C. H. 2006, A\&A, 652, 666

Tatulli, E., Millour, F., Chelli, A., et al. 2007, A\&A, 464, 29

Thiébaut, E. 2008, SPIE Proc., 7013, 70131I

Townes, C. H., Wishnow, E. H., Hale, D. D. S., \& Walp, B. 2009, ApJ, 697, L127

Tsuji, T. 2000a, ApJ, 538, 801

Tsuji, T. 2000b, ApJ, 540, L99

Tsuji, T. 2006, ApJ, 645, 1448

Verhoelst, T., Decin, L., Van Malderen, R., et al. 2006, A\&A, 447, 311 
A\&A 529, A163 (2011)

\section{Appendix A: Summary of AMBER observations}

Our AMBER observations of Betelgeuse and the calibrator Sirius are summarized in Table A.1.

Table A.1. Log of AMBER observations of Betelgeuse and the calibrator Sirius with the E0-G0-H0 (16-32-48 m) baseline configuration. Seeing is in the visible.

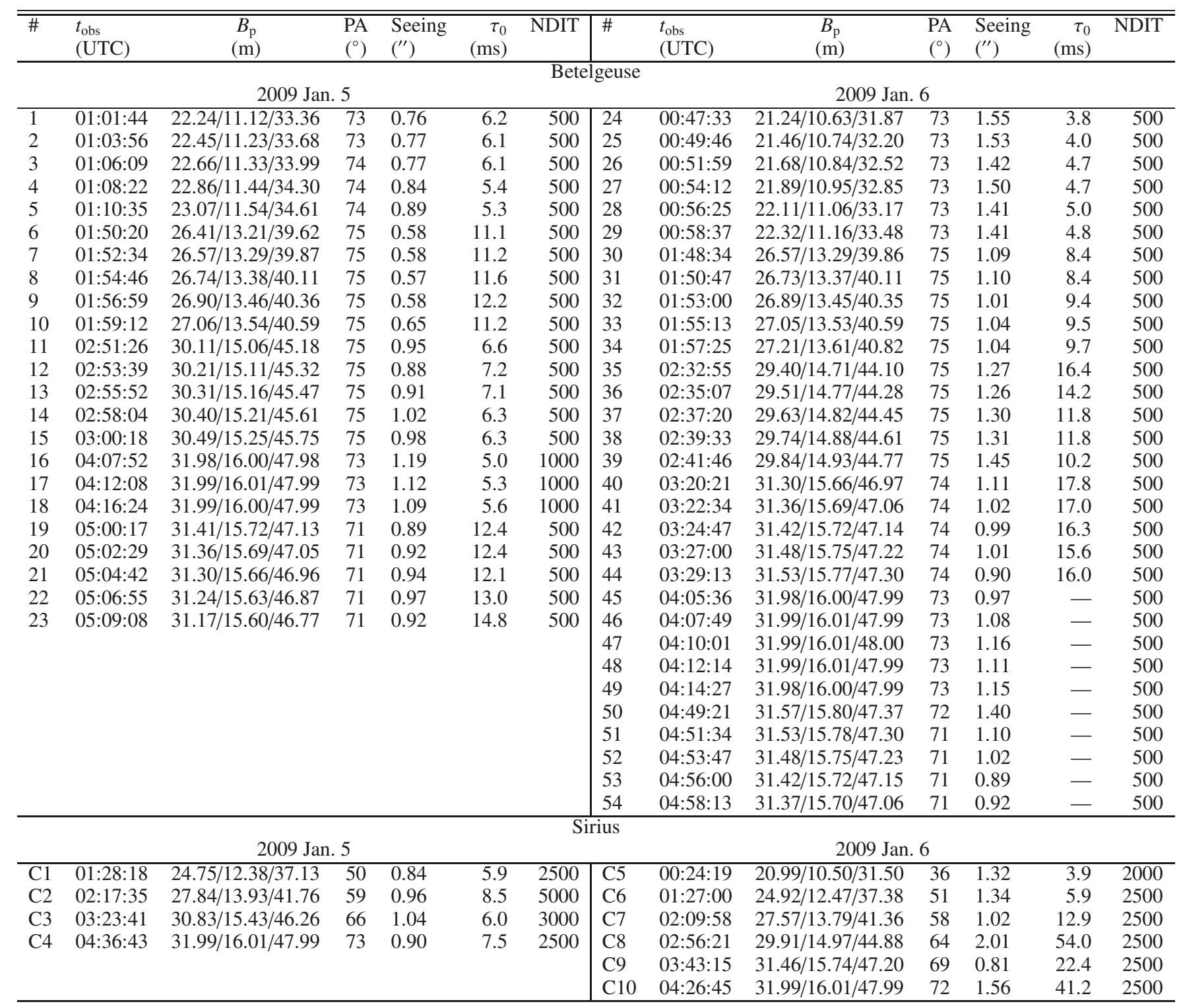




\section{Appendix B: Image reconstruction of simulated data}

Aperture synthesis imaging from optical/IR interferometric data depends on a number of parameters used in the image reconstruction process, such as the initial model, as well as the regularization scheme and prior, which represent a priori information about the object's intensity distribution. Therefore, it is important to carry out the image reconstruction for simulated images to derive the appropriate reconstruction parameters before we attempt the image reconstruction from observed data. From simulated images, we generate simulated interferometric data by sampling the visibilities and CPs at the same $u v$ points as interferometric observations. With the true images known for these simulated data, we can examine the appropriate reconstruction parameters that allow us to reconstruct the original images correctly.

Because MiRA is developed for two-dimensional image reconstruction, we took the following approach for the reconstruction of one-dimensional projection images: twodimensional image reconstruction was carried out using an appropriate initial model and regularization parameters as described below. The reconstructed two-dimensional image was convolved with the clean beam, which is represented by a twodimensional Gaussian with a $F W H M$ of $\lambda / B_{\max }=9.8$ mas, where $B_{\max }$ is the maximum baseline length of the data. The onedimensional projection image was obtained by integrating this convolved two-dimensional image in the direction perpendicular to the linear $u v$ coverage.

We generated two simulated images that represent possible surface patterns of Betelgeuse: a simple limb-darkened disk and a uniform disk with a bright spot, a dark spot, and an extended halo, as shown in Figs. B.1a and B.2a, respectively. For both cases, the stellar angular diameter was set to be 42.5 mas, which is the limb-darkened disk diameter derived from all continuum visibilities measured in 2009. The visibilities and CPs were computed from the simulated images at the same $u v$ points as our AMBER observations, using the program of one of the authors (K.-H. Hofmann). Noise was also added to the simulated visibilities and CPs to achieve SNRs similar to the AMBER data. We tested different initial models, priors, and regularization schemes to find out the appropriate parameter range to reconstruct the one-dimensional projection image of the simulated data correctly. It turned out that uniform disks with angular diameters between 34 and 50 mas serve as good initial models. The prior used in the present work is a smoothed uniform disk described as

$\operatorname{Pr}(r)=\frac{1}{\mathrm{e}^{\left(r-r_{\mathrm{p}}\right) / \varepsilon_{\mathrm{p}}}+1}$,

where $r$ is the radial coordinate in mas, and $r_{\mathrm{p}}$ and $\varepsilon_{\mathrm{p}}$ define the size and the smoothness of the edge $\left(\varepsilon_{\mathrm{p}} \rightarrow 0\right.$ corresponds to a uniform disk), respectively. The appropriate values for $r_{\mathrm{p}}$ and $\varepsilon_{\mathrm{p}}$ were found to be $10 \ldots 15$ (mas) and $2 \ldots 3$ (mas), respectively. Therefore, we used six different parameter sets for the image reconstruction of Betelgeuse by combining three diameters for the initial uniform-disk model $(34,42$, and 50 mas) and two different parameter sets for the prior $\left(\left(r_{\mathrm{p}}, \varepsilon_{\mathrm{p}}=(10,2)\right.\right.$ and $\left.(15,3)\right)$. The final images and their uncertainties were obtained by taking the average and standard deviation, respectively, from the results reconstructed with these six parameter sets. The regularization using the maximum entropy method turned out to be appropriate for our reconstruction. We started the reconstruction with a high degree of regularization ( $\mu=10^{5}$, see Thiébaut 2008, for the definition of $\mu$ ) and reduced it gradually by a factor of 10 after every 500 iterations until the reduced $\chi^{2}$ reaches $\sim 1$ or MiRA stops the iteration. These tests with the simulated data also confirm the validity of our approach to reconstruct one-dimensional projection images using the MiRA software for two-dimensional image reconstruction. 


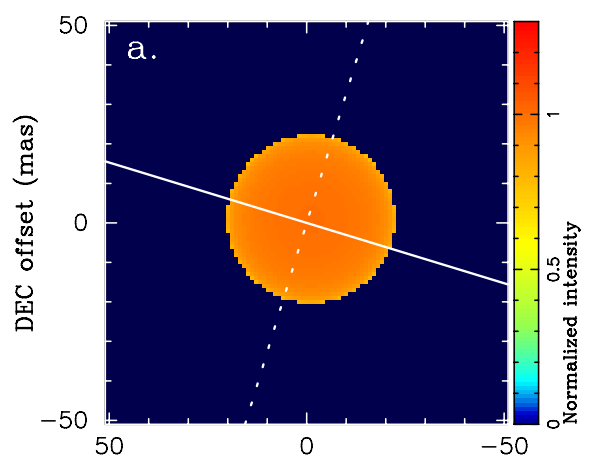

RA offset (mas)
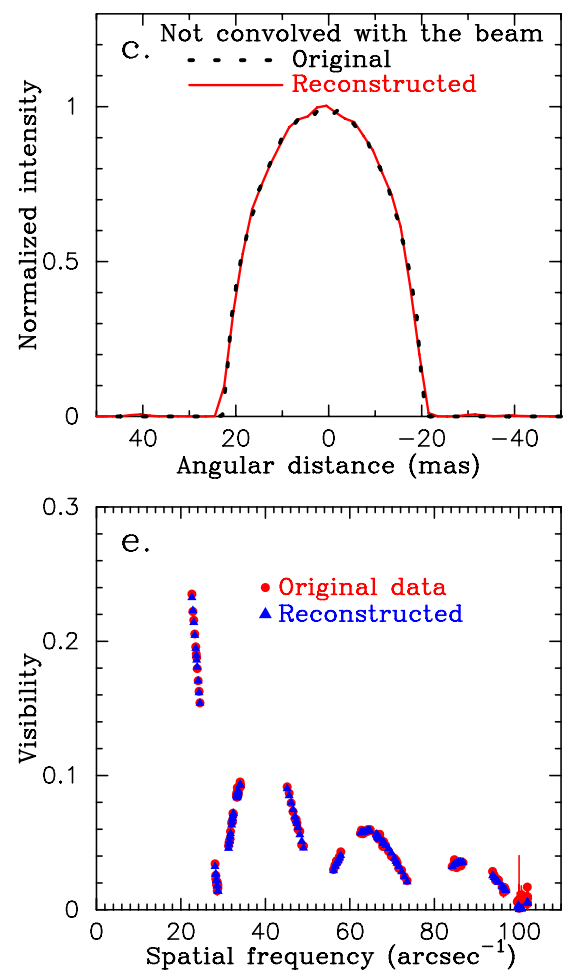

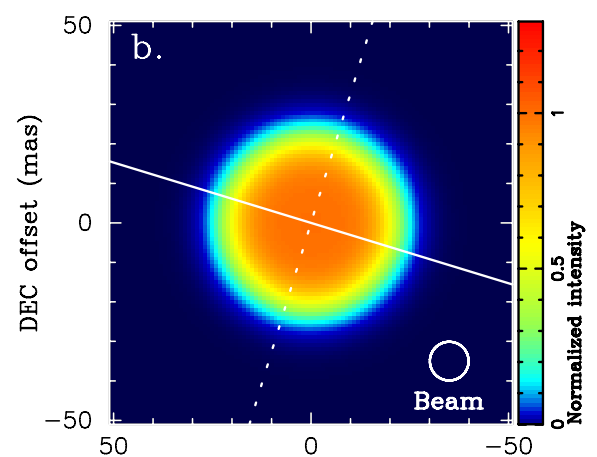

RA offset (mas)
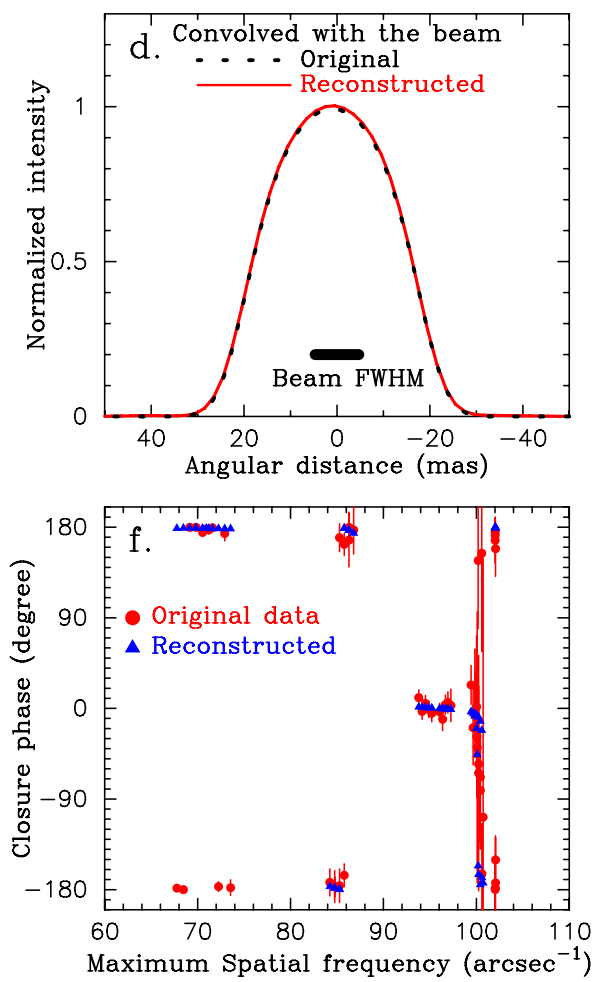

Fig. B.1. Image reconstruction of the simulated data for a limb-darkened disk with the parameters derived in Sect. 3.1. a) Original two-dimensional image of the simulated data. The solid line represents the orientation of the linear $u v$ coverage, while the dotted line represents the orientation perpendicular to it. b) Two-dimensional image of the simulated data convolved with the Gaussian beam with a $F W H M$ of 9.8 mas. c) Comparison between the original and reconstructed one-dimensional projection images before convolving with the Gaussian beam. The one-dimensional projection images are obtained by integrating the two-dimensional images in the direction shown by the dotted lines in the panels a) and b). d) Comparison between the original and reconstructed one-dimensional projection images convolved with the Gaussian beam with a $F W H M$ of 9.8 mas. e) The filled circles and triangles represent the visibilities from the original simulated data and the reconstructed image, respectively. f) The filled circles and triangles represent the CPs from the original simulated data and the reconstructed image, respectively. The abscissa is the spatial frequency of the longest baseline of each data set. 
K. Ohnaka et al.: Imaging the dynamical outer atmosphere of Betelgeuse in the CO first overtone lines
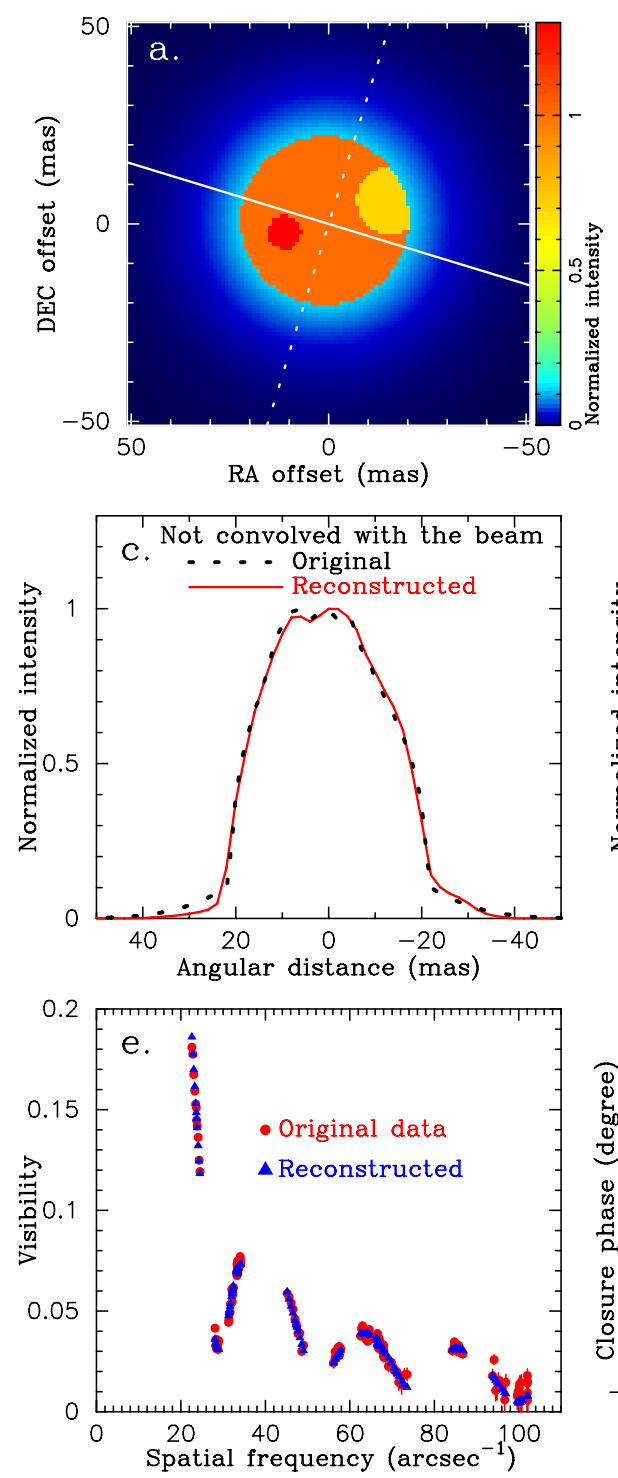
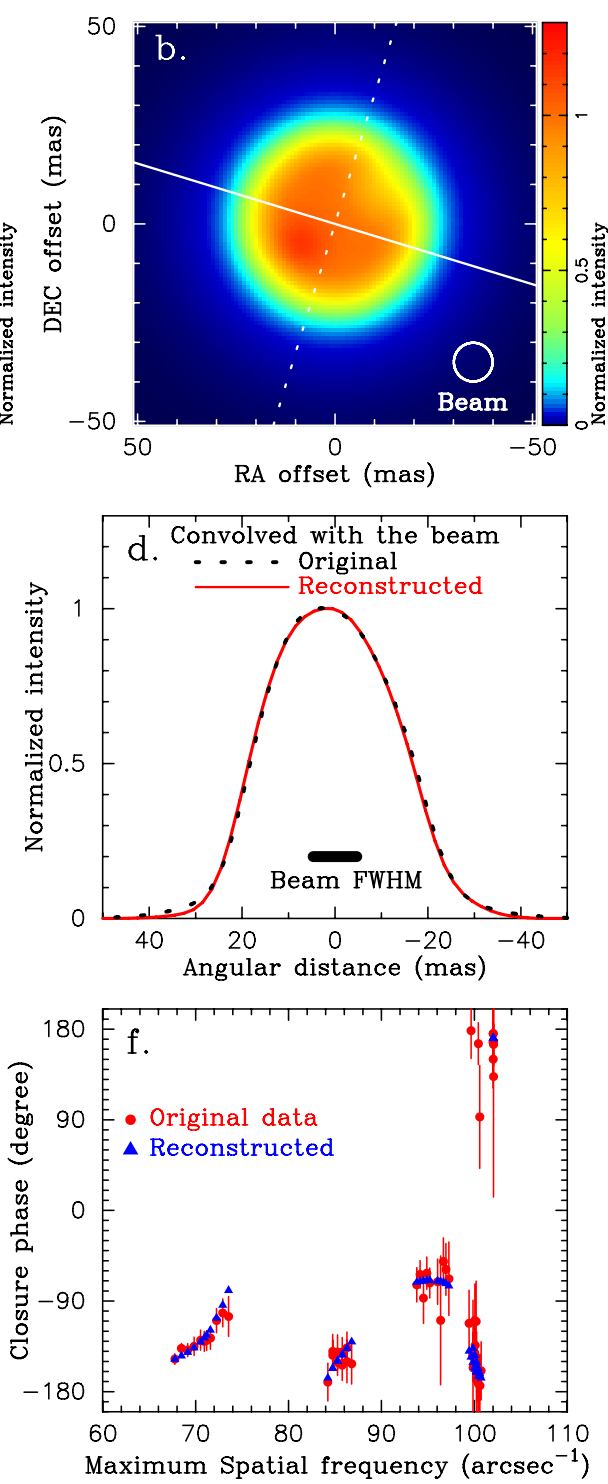

Fig. B.2. Image reconstruction of the simulated data for a uniform disk with a bright spot, a dark spot, and a halo shown in the same manner as in Fig. B.1. 


\section{Appendix C: Self-calibration imaging with differential phase}

Because the principle of the self-calibration technique using DP measurements is described in detail in Millour et al. (2011), we mention the actual procedure only briefly. Then we describe the modification we added to this technique to deal with an issue specific to the AMBER data of Betelgeuse.

The DP measured with AMBER at each $u v$ point contains information on the phase of the complex visibility function and roughly represents the difference between the phase in a spectral feature and that in the continuum. However, two pieces of information are lost because of the atmospheric turbulence: the absolute phase offset and the linear phase gradient with respect to wavenumber. We can derive this lost phase offset and gradient by a linear fit to the phase (as a function of wavenumber) from the reconstructed continuum images, if the image reconstruction in the continuum is reliable and not sensitive to the reconstruction parameters. This is indeed the case for our image reconstruction of Betelgeuse in the continuum, as discussed in Sect. 3.4. Therefore, the phase in the CO lines can be restored from the continuum phase interpolated at the line spectral channels and the DPs measured in the lines. The image reconstruction is carried out with the measured visibilities and CPs as well as the restored phase. This process can be iterated, but our experiments show that the reconstructed images do not change after the first iteration.

We added the following modification to the technique presented in Millour et al. (2011). When the phase offset and gradient are derived by a linear fit to the phase of the reconstructed images, we only use the continuum spectral channels below $2.293 \mu \mathrm{m}$ and those between the adjacent $\mathrm{CO}$ lines above $2.3 \mu \mathrm{m}$, instead of using the entire spectral channels, as Millour et al. (2011) did. The reason for this selection of the spectral channels is that the image reconstruction near the $\mathrm{CO}$ band head at $2.294 \mu \mathrm{m}$ is so uncertain owing to the poor SNR in the data binned with a spectral resolution of 6000 that the inclusion of the spectral channels near the band head in the linear fit hampers the reliable derivation of the phase offset and gradient.

The inclusion of only the selected continuum channels has the following consequence. If we denote the continuum phase from the reconstructed continuum image at a given baseline and at the $i$ th spectral channel as $\varphi_{\mathrm{c}}(i)$, the phase at the $i$ th spectral channel, $\varphi(i)$, is restored as

$\varphi(i)=\varphi_{\mathrm{c}}(i)+\mathrm{DP}(i)$,

where $\mathrm{DP}(i)$ represents the differential phase at the $i$ th spectral channel measured at the same baseline. At a continuum spectral channel denoted as $i_{\mathrm{c}}$, the restored phase $\varphi\left(i_{\mathrm{c}}\right)$ should be equal to the phase from the reconstructed continuum image $\varphi_{\mathrm{c}}\left(i_{\mathrm{c}}\right)$. This is fulfilled if the measured DP in the continuum is zero. However, the measured DPs show noticeable non-zero values in the continuum, as exemplarily shown in Figs. C.1a and b. The reason for the non-zero DPs in the continuum is that amdlib derives differential phase by a linear fit to the instantaneous phase at all spectral channels. Owing to the strong deviation of the phase in many CO lines from that in the continuum, this linear fit does not go through all the continuum points. Therefore, the non-zero DPs in the continuum spectral channels lead to a systematic error in the phase restored in the continuum, which affects the subsequent image reconstruction. We found out
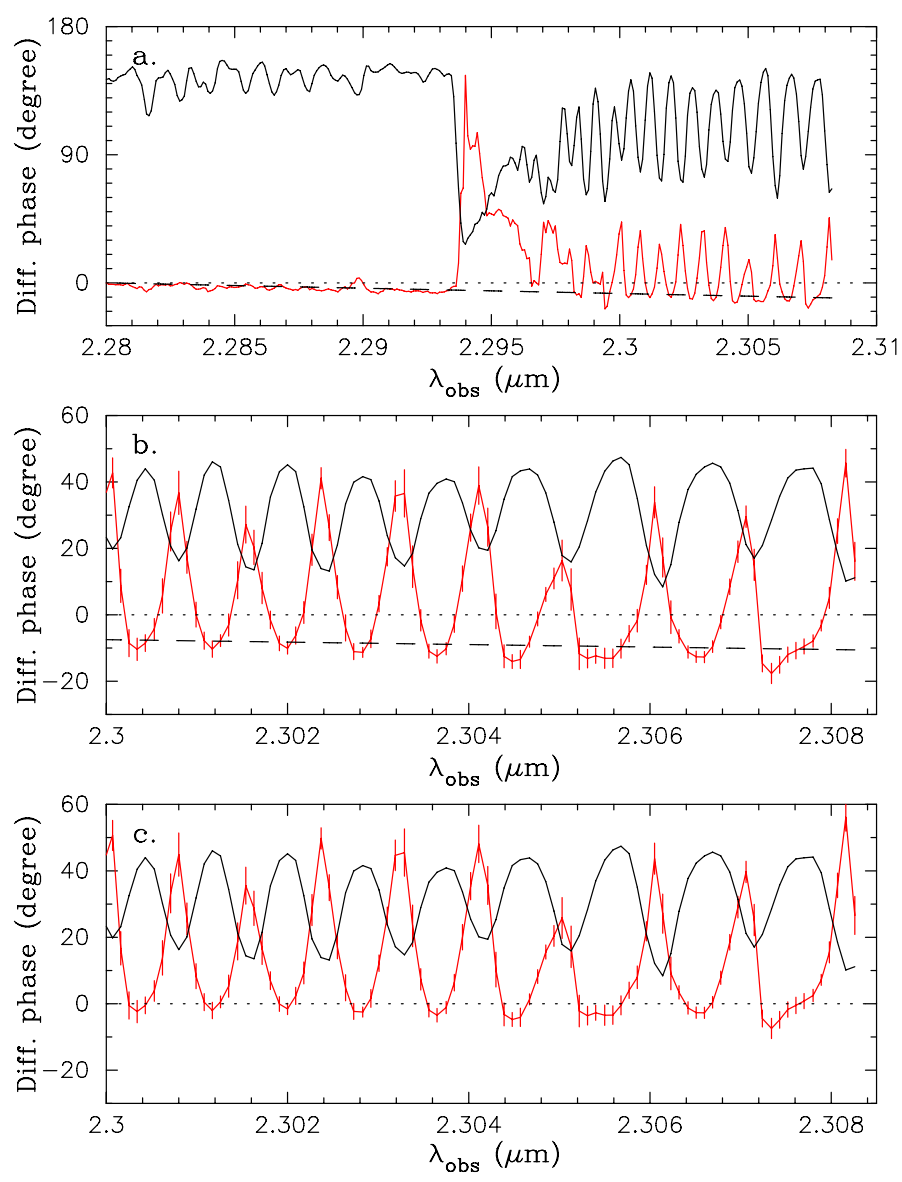

Fig. C.1. a) DP observed on the longest baseline in the data set \#9 $\left(B_{\mathrm{p}}=40.36 \mathrm{~m}\right)$ is plotted by the red solid line. The black line represents the scaled observed spectrum. The DP and spectrum are binned with a spectral resolution of 6000 . The dashed line represents the linear fit at the selected continuum points as described in Appendix C. DP $=0$ is shown by the dotted line. b) Enlarged view of panel a) for the CO lines. Note that the DP in the continuum points between the adjacent CO lines deviates from zero. c) DP after subtracting the linear fit to the continuum points as described in Appendix $\mathrm{C}$ is shown by the red solid line. The black line represents the scaled spectrum. The DP in the continuum points between the adjacent $\mathrm{CO}$ lines is now zero within the measurement errors.

that the continuum one-dimensional projection image reconstructed using the restored phase shows a systematic wavelength dependence from the shortest to the longest wavelength of the observed spectral range, which is not seen in the continuum images reconstructed from the visibilities and CPs alone.

It is necessary to use the same spectral channels in the linear fit to the phase for the derivation of DP and for the derivation of the phase offset and gradient. Therefore, we refitted the DP from amdlib with a linear function (with respect to wavenumber) at the same continuum points as used for the derivation of the phase offset and gradient (dashed lines in Figs. C.1a and b) and subtracted the fitted linear function from the observed DP. This procedure enforces the DP in the continuum spectral channels to zero within the measurement errors, as shown in Fig. C.1c. The phase was restored using this "refitted" DP. The continuum one-dimensional projection images reconstructed using the refitted DPs do not show the aforementioned systematic wavelength dependence, which proves the validity of our procedure. 


\section{Appendix D: Fit to the interferometric data with the reconstructed images}

Figures D.1 and D.2 show the fit to the observed interferometric data for the one-dimensional projection image reconstruction in the $\mathrm{CO}$ line and in the $\mathrm{CO}(2,0)$ band head shown in Figs. 5 and 6, respectively.
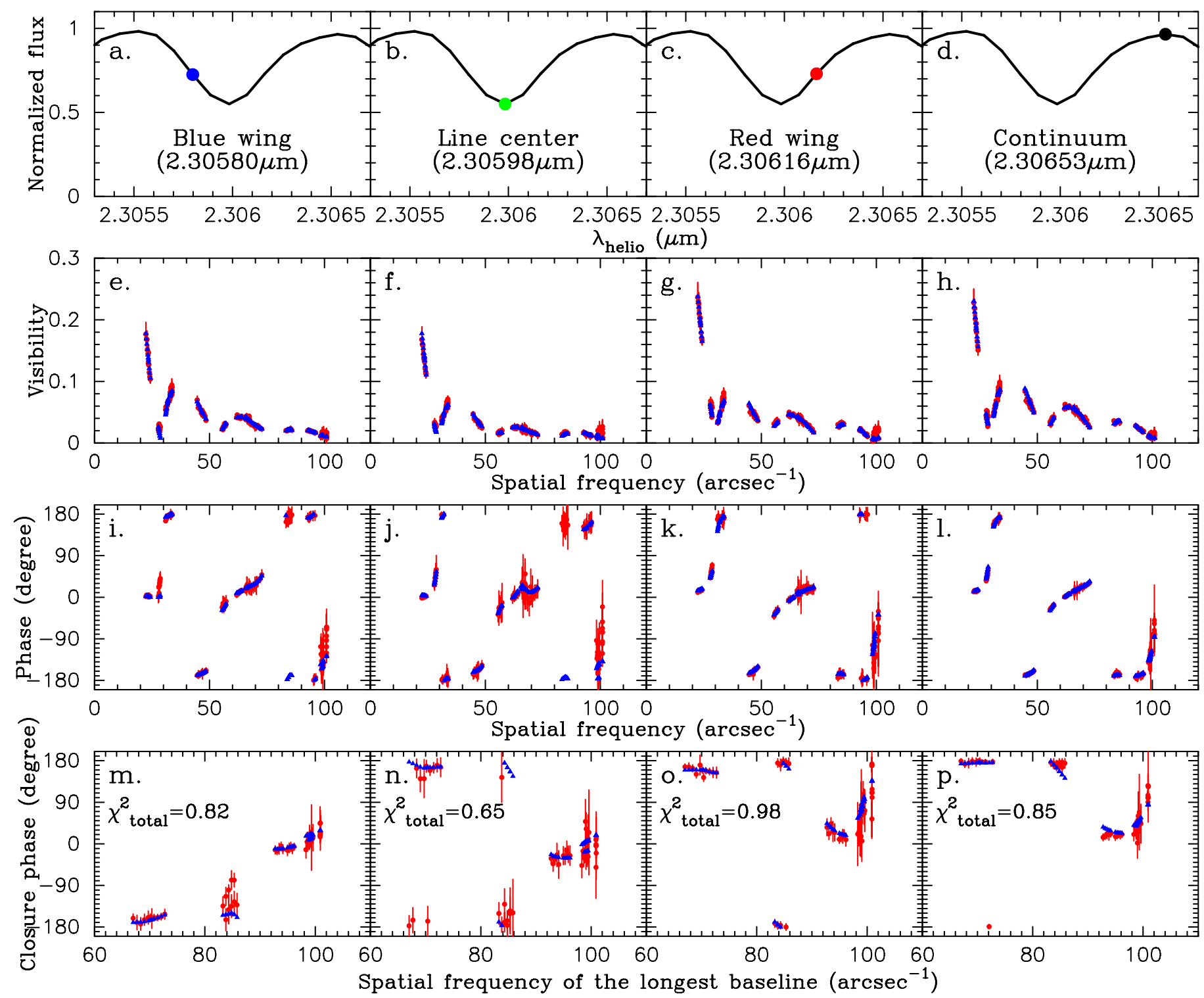

Fig. D.1. Comparison between the observed interferometric data and those from the one-dimensional projection image reconstruction for the CO line shown in Fig. 5. The first, second, third, and fourth columns show the comparison for the blue wing, line center, red wing, and continuum, respectively. The panels in the top row a)-d) show the observed CO line profile, and the filled circles denote the wavelength of the data shown in each column. In the remaining panels, the observed data are represented by the red circles, while the values from the image reconstruction are shown by the blue triangles. The reduced $\chi^{2}$ values for the fit including the complex visibilities, squared visibilities, and CPs are given in the panels $\mathbf{m})-\mathbf{p})$. 

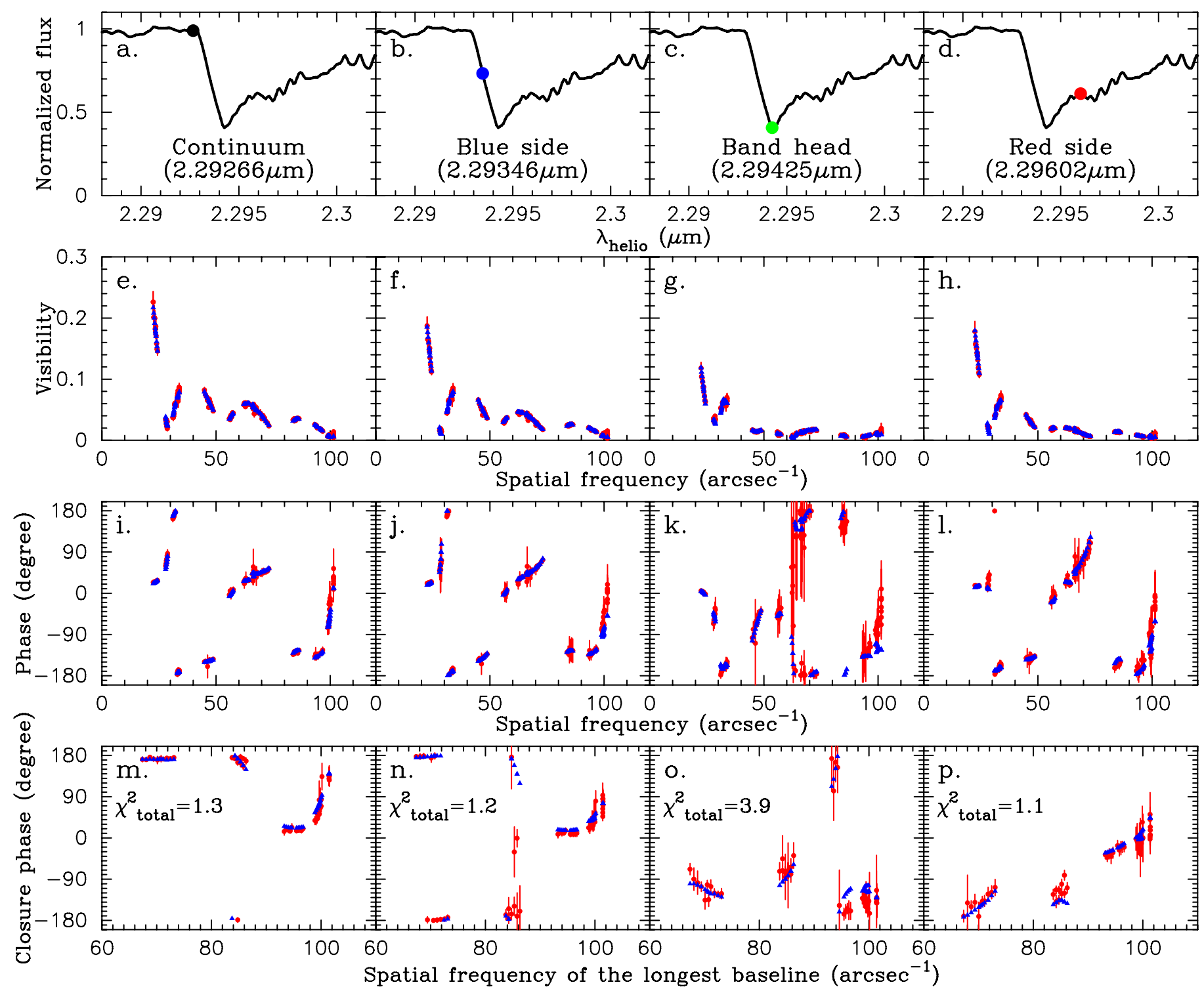

Fig. D.2. Comparison between the observed interferometric data and those from the one-dimensional projection image reconstruction near the CO $(2,0)$ band head shown in Fig. 6. The first, second, third, and fourth columns show the comparison for the continuum, blue side between the continuum and the band head, bottom of the band head, and red side of the band head, respectively. The panels in the top row a)-d) show the observed spectrum of the $\mathrm{CO}$ band head, and the filled circles denote the wavelength of the data shown in each column. In the remaining panels, the observed data are represented by the red circles, while the values from the image reconstruction are shown by the blue triangles. The reduced $\chi^{2}$ values for the fit including the complex visibilities, squared visibilities, and CPs are given in the panels $\mathbf{m}$ )-p). 